https://helda.helsinki.fi

\title{
Estimation of Viterbi path in Bayesian hidden Markov models
}

\author{
Lember, Juri
}

2019-08

Lember , J , Gasbarra , D , Koloydenko , A \& Kuljus , K 2019 , ' Estimation of Viterbi path in Bayesian hidden Markov models ' , Metron : rivista internazionale di statistica , vol. 77 , no. 2 , pp. 137-169 . https://doi.org/10.1007/s40300-019-00152-7

http://hdl.handle.net/10138/315294

https://doi.org/10.1007/s40300-019-00152-7

acceptedVersion

Downloaded from Helda, University of Helsinki institutional repository.

This is an electronic reprint of the original article.

This reprint may differ from the original in pagination and typographic detail.

Please cite the original version. 


\title{
Estimation of Viterbi path in Bayesian hidden Markov models
}

\author{
Jüri Lember ${ }^{1 *}$ Dario Gasbarra ${ }^{2}$, Alexey Koloydenko ${ }^{3}$ and Kristi Kuljus ${ }^{1}$ \\ May 14, 2019 \\ ${ }^{1}$ University of Tartu, Estonia; ${ }^{2}$ University of Helsinki, Finland; \\ ${ }^{3}$ Royal Holloway, University of London, UK
}

\begin{abstract}
The article studies different methods for estimating the Viterbi path in the Bayesian framework. The Viterbi path is an estimate of the underlying state path in hidden Markov models (HMMs), which has a maximum joint posterior probability. Hence it is also called the maximum a posteriori (MAP) path. For an HMM with given parameters, the Viterbi path can be easily found with the Viterbi algorithm. In the Bayesian framework the Viterbi algorithm is not applicable and several iterative methods can be used instead. We introduce a new EM-type algorithm for finding the MAP path and compare it with various other methods for finding the MAP path, including the variational Bayes approach and MCMC methods. Examples with simulated data are used to compare the performance of the methods. The main focus is on non-stochastic iterative methods and our results show that the best of those methods work as well or better than the best MCMC methods. Our results demonstrate that when the primary goal is segmentation, then it is more reasonable to perform segmentation directly by considering the transition and emission parameters as nuisance parameters.
\end{abstract}

Keywords: HMM, Bayes inference, MAP path, Viterbi algorithm, segmentation, EM, variational Bayes, simulated annealing

\section{Introduction and preliminaries}

Hidden Markov models (HMMs) are widely used in application areas including speech recognition, computational linguistics, computational molecular biology, and many more. Recently there has been a continuing interest to apply HMMs in the Bayesian framework, where model parameters are assumed to have a prior distribution. The Bayesian approach has the advantage that it allows researchers to incorporate their prior beliefs and information in the modeling process. However the Bayesian framework might heavily complicate the analysis, since a mixture of HMMs is not typically an HMM any more. Therefore the algorithms and methods valid for a single HMM might not be applicable in the Bayesian setup. For example, when several HMMs are mixed, the optimality principle no longer holds and dynamic programming algorithms such as the Viterbi algorithm and forward-backward algorithms do not work. Therefore finding the Viterbi path in the Bayesian framework is a difficult task where no simple solution exists.

Most of the literature on HMMs in the Bayesian framework (see, e.g. [6, 22, 27, 37, 34, 30, 9]) deals with MCMC methods [37, 6, 22, 34, 4]. When the goal is estimation of the underlying hidden state path

\footnotetext{
${ }^{*}$ Corresponding author, e-mail: jyri.lember@ut.ee
} 
for a given observation sequence which we refer to as segmentation (also terms decoding, denoising are used), various methods based on Gibbs sampling, for example simulated annealing, are often used. Note that simulated annealing works well only if the applied cooling schedule is correct and the number of sweeps large enough. In this article, we study and compare different non-stochastic methods for finding the Viterbi path in the Bayesian framework, because in comparison to MCMC methods non-stochastic methods are computationally less demanding. We introduce a new EM-type segmentation method which we call segmentation EM, and give an overview of other most commonly used non-stochastic segmentation methods. Unlike in the traditional EM algorithm, in the segmentation EM algorithm the hidden path is considered as the main parameter of interest. The performance of segmentation EM is compared with the other segmentation methods (including the variational Bayes approach and parameter EM estimation procedures) in numerical examples. According to our numerical examples, the segmentation EM method and a closely related method which we call segmentation $M M$, perform at least as well or better than MCMC methods. Moreover our empirical studies demonstrate that the direct Bayesian segmentation approach outperforms the commonly used parameters-first approach, where segmentation is performed after the parameters have been estimated.

Viterbi path estimation in the Bayesian framework has been studied and applied in speech tagging problems [14, 17, 12, 13]. An overview of Bayesian HMMs in speech tagging can be found in [8]. These papers study several methods for calculating the Viterbi path, including simulated annealing, variational Bayes, and also the parameters-first approach. The results of the studies are contradictory (see, e.g. [13]), showing that further research in this area is needed.

This article is organized as follows. In the rest of this section we introduce the problem of estimating the Viterbi path in the frequentist and Bayesian frameworks; at the end of the section the main objectives of the article will be summarized. Section 2 gives a brief overview of the methods and algorithms that we consider. Section 3 presents the results of numerical examples: first, in subsection 3.2, we consider the case where the emission parameters are known and transition parameters are unknown having Dirichlet prior distributions; then in subsection 3.3 we consider the case where the emission parameters are also unknown. In Section 4 the role of hyperparameters and their effect on segmentation results is discussed. Section 5 explains the relationships and similarities of the segmentation algorithms that we study. The formulae needed to apply the segmentation methods are presented in the Appendix.

\subsection{Segmentation with hidden Markov models}

Hidden Markov model. Consider a homogeneous Markov chain $Y^{n}:=Y_{1}, \ldots, Y_{n}$ with states $S=$ $\{1, \ldots, K\}$. Let $X^{n}:=X_{1}, \ldots, X_{n}$ be random variables taking values on $\mathcal{X}$ such that: 1) given $Y^{n}$, the random variables $\left\{X_{t}\right\}, t=1, \ldots, n$, are conditionally independent; 2) the distribution of $X_{t}$ depends on $Y^{n}$ only through $Y_{t}$. Since only $X^{n}$ is observed, the pair $\left(Y^{n}, X^{n}\right)$ is referred to as a hidden Markov model. Because the underlying Markov chain is homogeneous, the model is fully specified by the transition matrix $\mathbb{P}=\left(p_{l j}\right), l, j=1, \ldots, K$, initial probabilities $p_{0 k}, k=1, \ldots, K$, and emission distributions $P\left(X_{t} \in \cdot \mid Y_{t}=k\right), k=1, \ldots, K$. Thus there are two types of parameters in our model: transition parameters $\Theta_{t r}$ where $\theta_{t r} \in \Theta_{t r}$ specifies the transition matrix and the initial probability vector, and emission parameters $\Theta_{e m}$. Often the initial distribution is fixed or a function of the transition matrix. In this case any $\theta_{t r}$ can be identified with a transition matrix. The whole parameter space is given by $\Theta:=\Theta_{e m} \times \Theta_{t r}$. Without loss of generality we assume that all emission distributions have emission densities $f_{k}$ with respect to some common reference measure on $\mathcal{X}$. Typically, all emission densities are assumed to belong to the same parametric family $\mathcal{F}=\left\{f(\cdot \mid \theta): \theta \in \Theta_{\text {em }}\right\}$. Thus, for any state $k \in S$, there is a $\theta_{e m}^{k} \in \Theta_{e m}$ such that $f_{k}(\cdot)=f_{k}\left(\cdot \mid \theta_{e m}^{k}\right)$. For any realization $x^{n}$ of the random variables $X^{n}$ and 
for any realization $y^{n}$ of the Markov chain $Y^{n}$, let the joint likelihood of $\left(y^{n}, x^{n}\right)$ be denoted by $p\left(y^{n}, x^{n}\right)$. Similarly, $p\left(x^{n}\right)$ and $p\left(y^{n}\right)$ denote the marginal likelihoods of $p\left(y^{n}, x^{n}\right)$, and $p\left(x^{n} \mid y^{n}\right)$ and $p\left(y^{n} \mid x^{n}\right)$ stand for the conditional likelihoods. We assume that the length of the observation sequence is fixed and leave it from the notation. Thus, we denote by $x \in \mathcal{X}^{n}$ a vector $x^{n}$ of observations and by $y, s \in S^{n}$ state sequences. Also, $X$ and $Y$ stand for $X^{n}$ and $Y^{n}$, respectively, $p(y, x)$ is used instead of $p\left(y^{n}, x^{n}\right)$ and so on. To indicate a single entry of a vector $x$ or $s$, we use $x_{t}$ or $s_{t}, t=1, \ldots, n$. For any $t=1, \ldots, n, p_{t}$ is used for marginal probability, for example

$$
p_{t}\left(y_{t} \mid x\right)=P\left(Y_{t}=y_{t} \mid X=x\right)=\sum_{s: s_{t}=y_{t}} p(s \mid x) .
$$

Viterbi path. Suppose $\theta=\left(\theta_{t r}, \theta_{e m}\right) \in \Theta$ is given, that is both the transition and emission parameters are given. The segmentation problem consists of estimating the unobserved realization of the underlying Markov chain $Y$ given observations $X$. Formally, we are looking for a mapping $g: \mathcal{X}^{n} \rightarrow S^{n}$ called a classifier, that maps every sequence of observations into a state sequence. The best classifier $g$ is often defined via a loss function, for an overview of risk-based segmentation with HMMs based on loss functions, see $[20,26,41]$. The Viterbi path for given parameters $\theta$ is defined as a state path that maximizes the conditional probability $p(y \mid x, \theta)$. The solution of $\max _{y \in S^{n}} p(y \mid x, \theta)$ can be found by a dynamic programming algorithm called the Viterbi algorithm. For a given observation sequence $x$ and an HMM with initial probabilities $\left(p_{0 k}\right)$, transition matrix $\mathbb{P}=\left(p_{l j}\right)$ and emission densities $f_{k}(\cdot)(k, l, j \in S)$, the Viterbi algorithm is the following:

(1) for every $k \in S$, define $\delta_{1}(k):=p_{0 k} f_{k}\left(x_{1}\right)$;

(2) for $t=1, \ldots, n-1$ and $k \in S$ calculate

$$
\delta_{t+1}(k)=\max _{l \in S}\left(\delta_{t}(l) p_{l k}\right) f_{k}\left(x_{t+1}\right),
$$

and record

$$
l_{t}(k):=\arg \max _{j \in S} \delta_{t}(j) p_{j k}
$$

(3) find the Viterbi path $v$ by backtracking:

$$
v_{n}:=\arg \max _{k \in S} \delta_{n}(k), \quad v_{t}=l_{t}\left(v_{t+1}\right), \quad t=n-1, \ldots, 1 .
$$

Observe that the Viterbi path is not necessarily unique, any path $v=\arg \max _{y \in S^{n}} p(y \mid x, \theta)$ is called a Viterbi path. When emission distributions are continuous (like Gaussian as in the present paper), the Viterbi path is unique almost surely. Although the Viterbi path is not optimal for minimizing the expected number of classification errors, it is the most popular and most studied hidden path estimate in practice (see e.g. [33, 22, 5, 7]). The hidden path estimate that minimizes the expected number of classification errors is the so-called PMAP (pointwise maximum a posteriori) path which maximizes the $\operatorname{sum} \sum_{t=1}^{n} p_{t}\left(y_{t} \mid x, \theta\right)$. Since the sum can obviously be maximized termwise, the PMAP path $y$ is just a state path where $y_{t}, t=1, \ldots, n$, maximizes the marginal probability $p_{t}\left(y_{t} \mid x, \theta\right)$, and therefore the PMAP path can be found pointwise. Because of the pointwise optimization its posterior probability can be zero due to inadmissible transitions. The Viterbi and PMAP path are of different nature and for many models the difference between them can be rather big. For a discussion about the Viterbi, PMAP and related paths, see [21]. 


\section{$1.2 \quad$ Bayesian approach}

The Viterbi algorithm is applicable when the transition matrix as well as emission parameters are known. When this is not the case, the standard approach is to first estimate the parameters and then perform segmentation. This approach - parameters first, then segmentation - is also applicable in the Bayesian framework, where the parameters of an HMM are considered random. Indeed, one can find a Bayesian point estimate, typically the posterior mode $\hat{\theta}=\arg \max _{\theta} p(\theta \mid x)$, and then perform segmentation. However, if the primary goal is segmentation rather than parameter estimation, one can consider the true underlying path as the actual parameter of interest and the emission and transition parameters as nuisance parameters, and perform segmentation directly. Let us explain that approach more formally.

Bayesian Viterbi path. Let $\pi$ be a prior density in $\Theta$ with respect to a reference measure $d \theta$. For any $\theta=\left(\theta_{t r}, \theta_{\text {em }}\right)$ and for any pair $(x, y)$,

$$
p(x, y \mid \theta)=p\left(x \mid y, \theta_{e m}\right) p\left(y \mid \theta_{t r}\right), \quad p(y, x)=\int p(y, x \mid \theta) \pi(\theta) d \theta .
$$

It is important to note that although for any parameter set $\theta$ the measure $p(y, x \mid \theta)$ is a distribution of an HMM, then the measure $p(y, x)$ obtained after mixing is a distribution of a process that in general is not an HMM (sometimes called mixed-HMM, see [28, 29]). This complication is typical in the Bayesian setup and not specific to HMMs - a mixture of a product measure (the law of independent random variables) is not a product measure anymore, a mixture of Markov chains is not a Markov chain anymore, and so on. However, this circumstance complicates the whole analysis. As previously, the Viterbi path $v$ is defined as any state sequence $y \in S^{n}$ that maximizes the probability $p(y \mid x)$ over all state sequences: $v=\arg \max _{y \in S^{n}} p(y \mid x)$. As in the case of HMM, the Viterbi path is not necessarily unique, although in the case of continuous emissions it typically is. It might happen though that for many paths the probability $p(y \mid x)$ is very close to maximum, and even if these paths are not formally Viterbi paths (because they correspond to local and not to global maximums), they might often be outputs of the iterative algorithms considered in the article. This could seem disappointing at the first sight that the algorithms fail to find the global maximum, but since the conditional probabilities are very close to the maximum, these suboptimal paths could be considered as good substitutes of the Viterbi path.

As it is typical in Bayesian analysis, any Viterbi path $v$ is best only on average. For a given parameter $\theta$ generated from $\pi$, the path $v$ obviously does not need to maximize the probability $p(y \mid x, \theta)$, but it maximizes (over $y$ ) the average probability:

$$
p(y \mid x)=\frac{p(y, x)}{p(x)}=\frac{\int p(y, x \mid \theta) \pi(\theta) d \theta}{p(x)}=\frac{\int p(y \mid x, \theta) p(x \mid \theta) \pi(\theta) d \theta}{p(x)}=\int p(y \mid x, \theta) p(\theta \mid x) d \theta,
$$

where $p(\theta \mid x)$ is the posterior probability. When the observations $x$ are generated from a particular distribution with the true parameter $\theta^{*}$ and $x$ is sufficiently long, then according to the posterior consistency the posterior measure $p(\theta \mid x)$ is concentrated around $\theta^{*}$, and then the Bayesian approach should give more or less the same result as the parameter-first approach. Therefore, the Bayesian approach is more appealing when the sequence of observations $x$ is not very long. In particular, it might be a very reasonable choice in HMM pattern recognition setup when the training data consist of pairs $\left(x^{1}, y^{1}\right), \ldots,\left(x^{m}, y^{m}\right)$. For a training pair $\left(x^{j}, y^{j}\right)$ the observation sequence $x^{j}$ and the corresponding state sequence $y^{j}$ are assumed to be generated from an HMM with unknown parameter $\theta^{j}$. There is a target sequence $x$ whose Viterbi path needs to be estimated. Observe that in pattern recognition the Viterbi path $v$ is a common choice, 
because it minimizes the following expected loss

$$
v=\arg \min _{y} \sum_{y^{\prime}} L\left(y^{\prime}, y\right) p\left(y^{\prime} \mid x\right), \quad L\left(y^{\prime}, y\right)= \begin{cases}1, & \text { when } y=y^{\prime} \\ 0, & \text { else. }\end{cases}
$$

In this setup, for every $j$ the parameter estimate $\hat{\theta}^{j}$ can be found. When these estimates do not vary much, it is reasonable to believe that the true parameters $\theta^{j}$ are the same: $\theta^{j}=\theta^{*}$. The best one can do is to aggregate all estimates $\hat{\theta}^{j}$ into one estimate $\hat{\theta}$ which gives a reliable estimate of $\theta^{*}$, and use $\hat{\theta}$ to estimate the Viterbi path: $\hat{v}=\arg \max _{y} p(y \mid x, \hat{\theta})$. However, when the parameter estimates $\hat{\theta}^{j}$ vary a lot, it is reasonable to believe that the training data parameters $\theta^{j}$ are not the same, but rather constitute a sample from a prior distribution $\pi$. The prior $\pi$ could be chosen so that its variance (or mean, moments, hyperparameters) matches the variance (or mean, moments, hyperparameters) of the sample $\hat{\theta}^{1}, \ldots, \hat{\theta}^{m}$. Assuming that the true parameter is generated by a prior $\pi$, the best one can then do is to find the path that maximizes the average likelihood as in (1.2).

Prior distributions. In this article, we assume that the number of states $K$ as well as initial probabilities $p_{0 k}$ are known and uniform. It means that we shall not put any prior on $K$ and initial probabilities, and throughout the paper we take $p_{0 k}=1 / K, k=1, \ldots, K$. However, we shall put prior $\pi$ on the set of transition matrices and emission parameters. The prior $\pi$ is assumed to be such that emission and transition parameters are independent: $\pi(\theta)=\pi_{e m}\left(\theta_{e m}\right) \pi_{t r}\left(\theta_{t r}\right)$, where $\pi_{e m}$ and $\pi_{t r}$ are marginals. Then

$$
p(y, x, \theta)=p\left(y \mid \theta_{t r}\right) \pi_{t r}\left(\theta_{t r}\right) p\left(x \mid y, \theta_{e m}\right) \pi_{e m}\left(\theta_{e m}\right),
$$

with

$$
p\left(y \mid \theta_{t r}\right)=p_{0 y_{1}} \prod_{l j}\left(p_{l j}\left(\theta_{t r}\right)\right)^{n_{l j}(y)}, \quad p\left(x \mid y, \theta_{e m}\right)=\prod_{k=1}^{K} \prod_{t: y_{t}=k} f_{k}\left(x_{t} \mid \theta_{e m}^{k}\right),
$$

where $n_{l j}(y)$ denotes the number of transitions from state $l$ to state $j$ in the state sequence $y$. In particular, (1.3) ensures that for given $y$ and $x, p(y)$ depends on transition priors and $p(x \mid y)$ depends on emission priors only. The independence also implies that the posterior of the transition parameters depends only on $y: p\left(\theta_{t r} \mid x, y\right)=p\left(\theta_{t r} \mid y\right)$, and that $\theta_{e m}$ and $\theta_{t r}$ are independent under posterior measure:

$$
p(\theta \mid x, y)=p\left(\theta_{t r} \mid y\right) p\left(\theta_{e m} \mid y, x\right) .
$$

We consider the case where emission parameters are componentwise independent, that is $\pi_{e m}\left(\theta_{e m}\right)=$ $\pi_{e m}^{1}\left(\theta_{e m}^{1}\right) \cdots \pi_{e m}^{K}\left(\theta_{e m}^{K}\right)$ for $\theta_{e m}=\left(\theta_{e m}^{1}, \ldots, \theta_{e m}^{K}\right)$, which implies the independence under posterior:

$$
p\left(\theta_{e m} \mid x, y\right)=\prod_{k=1}^{K} p\left(\theta_{e m}^{k} \mid x, y\right) .
$$

Typically transition parameters are the transition probabilities, that is $p_{l j}\left(\theta_{t r}\right)=p_{l j}$. The standard approach in this case is to model all the rows of a transition matrix $\mathbb{P}=\left(p_{l j}\right)$ independently with the $l$-th row having a Dirichlet prior $\operatorname{Dir}\left(\alpha_{l 1}, \ldots, \alpha_{l K}\right)$, see e.g. [22, 6, 27, 9, 16, 14]. Thus,

$$
\pi_{t r}(\mathbb{P})=\pi_{t r}\left(p_{11}, \ldots, p_{1 K}\right) \pi_{t r}\left(p_{21}, \ldots, p_{2 K}\right) \cdots \pi_{t r}\left(p_{K 1}, \ldots, p_{K K}\right) \propto \prod_{l j} p_{l j}^{\alpha_{l j}-1}
$$


provided $\left(p_{l 1}, \ldots, p_{l K}\right) \in \mathbb{S}_{K}$, where $\mathbb{S}_{K}$ is a unit simplex. Since the rows are independent under the prior, they are also independent under the posterior, so that for a given path $y$, the $l$-th row has a Dirichlet distribution:

$$
p\left(\left(p_{l 1}, \ldots, p_{l K}\right) \mid y\right) \sim \operatorname{Dir}\left(\alpha_{l 1}+n_{l 1}(y), \ldots, \alpha_{l K}+n_{l K}(y)\right) .
$$

Let $n_{l}(y)=\sum_{j} n_{l j}(y)$ and $\alpha_{l}=\sum_{j} \alpha_{l j}$. Under a Dirichlet prior, the marginal probability of any path $y$ can be calculated as (see e.g. (19) in [9])

$$
p(y)=\int p\left(y \mid \theta_{t r}\right) \pi_{t r}\left(\theta_{t r}\right) d \theta_{t r}=p_{0 y_{1}} \prod_{l} \frac{\Gamma\left(\alpha_{l}\right)}{\Gamma\left(\alpha_{l}+n_{l}(y)\right)} \prod_{j} \frac{\Gamma\left(\alpha_{l j}+n_{l j}(y)\right)}{\Gamma\left(\alpha_{l j}\right)} .
$$

As is common in the case of Dirichlet priors (see e.g. [16]), we will use the factorization $\alpha_{l j}=M q_{l j}$, where $Q=\left(q_{l j}\right)$ is a transition matrix and $M>0$ can be regarded as the precision parameter. Thus, $Q$ postulates our belief about the general form of the transition matrix and $M$ shows how strongly we believe in it: the bigger $M$, the smaller the variance of $p_{l j}$.

\subsection{Objectives of the article}

The main goals of the article are the following:

- To give a brief overview of the most commonly used Bayesian segmentation methods (segmentation performed with Bayesian parameter estimates, segmentation MM, variational Bayes, iterative conditional mode, and simulated annealing) and study their performance. We present the general ideas behind the methods and derive the formulae needed for applying the methods. Although in general the methods mentioned above are well known, we believe that in the context of segmentation in Bayesian HMMs the methods are not so well studied and understood. The contradictory results in the speech tagging literature mentioned in the introduction are evidence of that. Therefore, a comparative study of these segmentation methods together with the necessary formulae might clarify the picture and help practitioners.

- To introduce the segmentation EM method and study its performance in comparison to the Bayesian segmentation methods mentioned above. Segmentation EM is a standard EM method where the path $y$ is considered as the main parameter of interest and $\theta$ as a nuisance parameter. Application of the segmentation EM algorithm depends very much on the particular model studied. For example, to apply it to the so-called triplet Markov models, see e.g. [15, 10, 23], some additional assumptions are needed. Similarly it is not clear how to apply segmentation EM in HMMs with infinite state spaces (hierarchical Dirichlet processes). Thus our main message concerning the segmentation EM algorithm is that in the case of HMMs the segmentation EM approach is applicable (at least for the priors considered in the paper) and works well. Among all the non-stochastic methods we consider, segmentation EM is the only one that iteratively maximizes $p(y \mid x)$, and therefore it is theoretically justified and recommended.

- To compare the performance of all the methods and to show that in Bayesian segmentation, the best non-stochastic iterative methods perform at least as well as MCMC methods such as simulated annealing, while at the same time being computationally faster and less demanding. 


\section{Bayesian segmentation methods}

\subsection{Segmentation EM}

Since our goal is to find a state sequence that maximizes $p(y \mid x)$, the main parameter of interest is the hidden path rather than the model parameters $\theta$. Therefore it is natural to change their roles in the EM procedure in order to maximize $p(y \mid x)$. Indeed - in the traditional Bayesian EM approach the objective is to maximize the posterior probability of parameters $p(\theta \mid x)$, and then $y$ is considered as the latent (or nuisance) parameter and integrated out. In our setup, the objective is to maximize $p(y \mid x)$, thus $\theta$ is considered as the latent (nuisance) parameter and integrated out.

We start with an initial sequence $y^{(0)}$ and then update the state sequences according to the following rule:

$$
y^{(i+1)}=\arg \max _{y} \int \ln p(y, \theta \mid x) p\left(\theta \mid y^{(i)}, x\right) d \theta=\arg \max _{y} \int \ln p(y, x \mid \theta) p\left(\theta \mid y^{(i)}, x\right) d \theta .
$$

Every iteration step increases the probability $p(y \mid x)$ and the algorithm stops when there are no further changes in the estimated state sequence. We call this procedure segmentation EM, the output is denoted by $\hat{v}_{\mathrm{sEM}}$.

Lemma 2.1. Every iteration step in the segmentation EM procedure increases the posterior probability: $p\left(y^{(i+1)} \mid x\right) \geq p\left(y^{(i)} \mid x\right)$. Furthermore, the objective function in (2.1) can be maximized with the Viterbi algorithm by considering the matrix $\left(u_{l j}^{(i)}\right)$ and the functions $h_{k}^{(i)}$ as the transition and emission parameters, where

$$
u_{l j}^{(i)}:=\exp \left[\int \ln p_{l j}\left(\theta_{t r}\right) p\left(\theta_{t r} \mid y^{(i)}\right) d \theta_{t r}\right], \quad h_{k}^{(i)}\left(x_{t}\right):=\exp \left[\int \ln f_{k}\left(x_{t} \mid \theta_{e m}^{k}\right) p\left(\theta_{e m}^{k} \mid y^{(i)}, x\right) d \theta_{e m}^{k}\right] .
$$

Observe that $u_{l j}^{(i)}$ and $h_{k}^{(i)}$ in (2.2) depend on iteration $i$. For the sake of simplicity we will suppress $(i)$ in the notation and write $u_{l j}$ and $h_{k}$ in the rest of this subsection.

Proof. It is well known that the standard EM algorithm increases the likelihood at every iteration step (see, e.g. [31, 6]). Change the roles of $\theta$ and $y$ to obtain $p\left(y^{(i+1)} \mid x\right) \geq p\left(y^{(i)} \mid x\right)$. To see that $y^{(i+1)}$ can be found with the Viterbi algorithm, note that by (1.1), (1.4) and (1.5) we have

$$
\begin{aligned}
& \int \ln p(y, x \mid \theta) p\left(\theta \mid y^{(i)}, x\right) d \theta=\int \ln p\left(y \mid \theta_{t r}\right) p\left(\theta_{t r} \mid y^{(i)}\right) d \theta_{t r}+\int \ln p\left(x \mid y, \theta_{e m}\right) p\left(\theta_{e m} \mid y^{(i)}, x\right) d \theta_{e m} \\
& =\ln p_{0 y_{1}}+\sum_{l, j \in S} n_{l j}(y) \int \ln p_{l j}\left(\theta_{t r}\right) p\left(\theta_{t r} \mid y^{(i)}\right) d \theta_{t r}+\sum_{k=1}^{K} \sum_{t: y_{t}=k} \int \ln f_{k}\left(x_{t} \mid \theta_{e m}^{k}\right) p\left(\theta_{e m}^{k} \mid y^{(i)}, x\right) d \theta_{e m}^{k} \\
& =\ln p_{0 y_{1}}+\sum_{l, j \in S} n_{l j}(y) \ln u_{l j}+\sum_{k=1}^{K} \sum_{t: y_{t}=k} \ln h_{k}\left(x_{t}\right) .
\end{aligned}
$$

Thus, the objective function is in the form of $\ln p(y, x)$ of an HMM with 'transition matrix' $\left(u_{l j}\right)$ and 'emission densities' $h_{k}$. By Jensen's inequality we know that the rows of $\left(u_{l j}\right)$ do not sum up to one, thus $\left(u_{l j}\right)$ is not a transition matrix. Similarly, the functions $h_{k}$ do not integrate to one, thus the functions $h_{k}$ are not probability densities. However, the Viterbi algorithm can still be applied to find the path with maximum probability. To see that, note first that the functions $h_{k}$ enter into the Viterbi algorithm only via values $h_{k}\left(x_{t}\right)$, so it really does not matter whether they integrate to one or not. Similarly, the optimality principle - if a maximum probability path passes state $k$ at time $m$, the first $m$ elements of 
that path must form a maximum likelihood path amongst those paths that end in state $k$ at time $m$ does not depend on whether the probabilities sum up to one or not. If the optimality principle holds, then the Viterbi algorithm as a dynamic programming algorithm finds the maximum probability path.

The fact that the Viterbi algorithm can be applied for maximizing $\ln p(y, x)$ makes the segmentation EM possible as soon as $\left(u_{l j}\right)$ and $h_{k}\left(x_{t}\right)$ can be calculated. In our case with Dirichlet priors for the transition parameters the posterior measure $p\left(\theta_{t r} \mid y\right)$ is the product of the row posteriors, and the posterior of the $l$-th row is $\operatorname{Dir}\left(\alpha_{l 1}+n_{l 1}(y), \ldots, \alpha_{l K}+n_{l K}(y)\right)$. Then

$$
p_{l j} \sim \operatorname{Be}\left(\alpha_{l j}+n_{l j}(y), \alpha_{l}+n_{l}(y)-\alpha_{l j}-n_{l j}(y)\right) .
$$

It is known that when $X \sim \operatorname{Be}(\alpha, \beta)$, then $E(\ln X)=\psi(\alpha)-\psi(\alpha+\beta)$, where $\psi$ is the digamma function. Thus, for any sequence $y$, the quantities $u_{l j}$ can be calculated with the following formula:

$$
\ln u_{l j}(y)=\int \ln p_{l j}\left(\theta_{t r}\right) p\left(\theta_{t r} \mid y\right) d \theta_{t r}=\psi\left(\alpha_{l j}+n_{l j}(y)\right)-\psi\left(\alpha_{l}+n_{l}(y)\right) .
$$

Computing $h_{k}$ depends on the family of emission densities. If emission distributions belong to an exponential family, that is

$$
f\left(x \mid \theta_{e m}\right)=\exp \left[\theta_{e m}^{t} T(x)+A\left(\theta_{e m}\right)+B(x)\right],
$$

then calculation of $h_{k}(x)$ reduces to evaluating the moments

$$
\int \theta_{e m} p\left(\theta_{e m} \mid y, x\right) d \theta_{e m}, \quad \int A\left(\theta_{e m}\right) p\left(\theta_{e m} \mid y, x\right) d \theta_{e m}
$$

For conjugate priors, this kind of integration is often feasible.

\subsection{Other segmentation methods}

Segmentation MM. The segmentation MM algorithm is just like the segmentation EM algorithm, except that the expectation step is replaced by the maximization step. We start with an initial path $y^{(0)}$. Then, given $y^{(i)}$, find

$$
\theta^{(i+1)}=\arg \max _{\theta} p\left(\theta \mid y^{(i)}, x\right), \quad y^{(i+1)}=\arg \max _{y} p\left(y \mid \theta^{(i+1)}, x\right) .
$$

The algorithm converges when there are no changes in the two consecutive path estimates. Every iteration step increases the joint likelihood, that is

$$
p\left(y^{(i+1)}, \theta^{(i+1)} \mid x\right) \geq p\left(y^{(i)}, \theta^{(i+1)} \mid x\right) \geq p\left(y^{(i)}, \theta^{(i)} \mid x\right),
$$

but the objective function $p(y \mid x)$ is not guaranteed to increase. In the context of parameter estimation in the non-Bayesian setting (that is when the prior is non-informative) this algorithm is sometimes called the Viterbi training [26, 24, 19, 25] or classification EM [36, 35]. It should move on faster than segmentation EM. The advantage of the segmentation MM procedure over the segmentation EM procedure is that it does not require calculation of $u_{l j}$ and $h_{k}$. For given $\theta^{(i)}$ the path $y^{(i)}$ can be found by the standard Viterbi algorithm, and $\theta^{(i+1)}$ is just the posterior mode; in our case the mode for the emission and transition parameters can be calculated separately due to independence: $\theta^{(i+1)}=\left(\theta_{t r}^{(i+1)}, \theta_{e m}^{(i+1)}\right)$, where

$$
\theta_{t r}^{(i+1)}=\arg \max _{\theta_{t r}} p\left(\theta_{t r} \mid y^{(i)}\right), \quad \theta_{e m}^{(i+1)}=\arg \max _{\theta_{e m}} p\left(\theta_{e m} \mid y^{(i)}, x\right) .
$$


Bayesian EM. The parameters-first approach in segmentation consists of estimating the unknown model parameters first and then performing segmentation. The most common parameter estimate in the Bayesian setup is the MAP estimate defined as

$$
\hat{\theta}=\arg \max _{\theta} p(\theta \mid x)=\arg \max _{\theta} p(x \mid \theta) \pi(\theta) .
$$

The standard method for calculating $\hat{\theta}$ is the EM algorithm [31, 22]. The EM procedure in the Bayesian setup starts with an initial parameter $\theta^{(0)}$ and updates the parameters iteratively as follows:

$$
\theta^{(i+1)}=\arg \max _{\theta} \sum_{y} \ln p(y, \theta \mid x) p\left(y \mid \theta^{(i)}, x\right)=\arg \max _{\theta}\left[\sum_{y} \ln p(y, x \mid \theta) p\left(y \mid \theta^{(i)}, x\right)+\ln \pi(\theta)\right] .
$$

Every iteration increases the posterior probability, that is $p\left(\theta^{(i+1)} \mid x\right) \geq p\left(\theta^{(i)} \mid x\right)$. We call this estimation procedure Bayesian EM and denote the resulting parameter estimate by $\hat{\theta}_{\mathrm{B}(\mathrm{EM})}$. The EM procedure in the non-Bayesian setup is the same, except that $\ln \pi(\theta)$ is missing on the right hand side of (2.4). This procedure will be called standard EM and the output of the procedure will be denoted by $\hat{\theta}_{\mathrm{EM}}$. Thus, the standard EM algorithm can be considered as a special case of the Bayesian EM algorithm with a non-informative prior $(\ln \pi(\theta)=$ const $)$. In the case of Dirichlet transition priors, noninformative priors correspond to the case $\alpha_{l j}=1$. The Viterbi path estimates $\hat{v}_{\mathrm{B}(\mathrm{EM})}$ and $\hat{v}_{\mathrm{EM}}$ are obtained by applying the Viterbi algorithm with the respective parameter estimates: $\hat{v}_{\mathrm{B}(\mathrm{EM})}:=\arg \max _{y} p\left(y \mid x, \hat{\theta}_{\mathrm{B}(\mathrm{EM})}\right), \hat{v}_{\mathrm{EM}}:=$ $\arg \max _{y} p\left(y \mid x, \hat{\theta}_{\mathrm{EM}}\right)$.

Variational Bayes approach. The idea behind the variational Bayes (VB) approach (see, e.g. [39, $38,11,18,1,2,5])$ is to approximate the posterior $p(\theta, y \mid x)$ with a product $\hat{q}_{\theta}(\theta) \hat{q}_{Y}(y)$, where $\hat{q}_{\theta}$ and $\hat{q}_{Y}$ are probability measures on the parameter space and $S^{n}$ that minimize the Kullback-Leibler divergence $D\left(q_{\theta} \times q_{Y} \| p(\theta, y \mid x)\right)$ over all product measures $q_{\theta} \times q_{Y}$, that is

$$
\hat{q}_{\theta} \times \hat{q}_{Y}=\arg \inf _{q_{\theta} \times q_{Y}} D\left(q_{\theta} \times q_{Y} \| p(\theta, y \mid x)\right) .
$$

It is known that the measures $\hat{q}_{\theta}$ and $\hat{q}_{Y}$ satisfy the equations

$$
\begin{aligned}
& \ln \hat{q}_{\theta}(\theta)=c_{1}+\int \ln p(\theta, y \mid x) \hat{q}_{Y}(d y)=c_{1}+\sum_{y} \ln p(\theta, y \mid x) \hat{q}_{Y}(y), \\
& \ln \hat{q}_{Y}(y)=c_{2}+\int \ln p(\theta, y \mid x) \hat{q}_{\theta}(d \theta),
\end{aligned}
$$

where $c_{1}$ and $c_{2}$ are constants. This suggests the following iterative algorithm for calculating $\hat{q}_{\theta}(\theta)$ and $\hat{q}_{Y}(y)$. Start with an initial sequence $y^{(0)}$ and take $q_{Y}^{(0)}=\delta_{y^{(0)}}$. Given $q_{Y}^{(i)}$, update the measures as

$$
\begin{aligned}
& \ln q_{\theta}^{(i+1)}(\theta)=c_{1}^{(i+1)}+\sum_{y} \ln p(\theta, y \mid x) q_{Y}^{(i)}(y), \\
& \ln q_{Y}^{(i+1)}(y)=c_{2}^{(i+1)}+\int \ln p(\theta, y \mid x) q_{\theta}^{(i+1)}(d \theta) .
\end{aligned}
$$

In [1], the algorithm is called variational Bayes EM and it is argued (Theorem 2.1) that it decreases the Kullback-Leibler divergence in the following sense:

$$
D\left(q_{\theta}^{(i)} \times q_{Y}^{(i)} \| p(\theta, y \mid x)\right) \geq D\left(q_{\theta}^{(i+1)} \times q_{Y}^{(i)} \| p(\theta, y \mid x)\right) \geq D\left(q_{\theta}^{(i+1)} \times q_{Y}^{(i+1)} \| p(\theta, y \mid x)\right) .
$$


Suppose the VB algorithm described above has converged and its final output is $\hat{q}_{\theta} \times \hat{q}_{Y}$. Then $\hat{q}_{Y}$ is taken as the approximation of $p(y \mid x)$ and the Viterbi path estimate $\hat{v}_{\mathrm{VB}}$ is obtained as $\hat{v}_{\mathrm{VB}}:=\arg \max _{y} \hat{q}_{Y}(y)$.

Applying the variational Bayes method for estimating the Viterbi path is certainly not a trivial task. All the formulae needed for updating $q_{Y}^{(i+1)}$ and $q_{\theta}^{(i+1)}$ with explanations about technical details are presented in the Appendix.

Simulated annealing. Let $1 \leq \beta_{1}<\beta_{2} \ldots<\beta_{r}$ be a cooling schedule. Since direct sampling from distribution $p_{\beta}(y \mid x) \propto p^{\beta}(y \mid x)$ is not possible, for every $\beta$ we sample $y_{\beta}^{(1)}, \theta_{\beta}^{(1)}, y_{\beta}^{(2)}, \theta_{\beta}^{(2)}, \ldots, y_{\beta}^{\left(n_{\beta}\right)}$ alternately from a probability measure $p_{\beta}(\theta, y \mid x) \propto p(\theta, y \mid x)^{\beta}$ in the acceptance-rejection sense as follows. For given $\beta$ and path $y^{(i)}$, generate the parameter $\theta^{(i)}$ from the distribution $p_{\beta}\left(\theta \mid y^{(i)}, x\right) \propto p\left(\theta \mid y^{(i)}, x\right)^{\beta}$. Then, given $\theta^{(i)}$, generate a path $y$ from $p_{\beta}\left(y \mid \theta^{(i)}, x\right) \propto p\left(y \mid \theta^{(i)}, x\right)^{\beta}$. The generated path $y$ will be accepted as $y^{(i+1)}$ with the probability

$$
\frac{p(y \mid x)^{\beta} /\left[p_{\beta}\left(y \mid \theta^{(i)}, x\right) p_{\beta}\left(\theta^{(i)} \mid y^{(i)}, x\right)\right]}{p\left(y^{(i)} \mid x\right)^{\beta} /\left[p_{\beta}\left(y^{(i)} \mid \theta^{(i)}, x\right) p_{\beta}\left(\theta^{(i)} \mid y, x\right)\right]} \wedge 1=\frac{p(y \mid x)^{\beta} / p_{\beta}(y, x)}{p\left(y^{(i)} \mid x\right)^{\beta} / p_{\beta}\left(y^{(i)}, x\right)} \wedge 1,
$$

where $J_{\beta}\left(y \mid y^{(i)}\right)=p_{\beta}\left(y \mid \theta^{(i)}, x\right) p_{\beta}\left(\theta^{(i)} \mid y^{(i)}, x\right)$ is the proposal distribution and $p_{\beta}(y, x) \propto \int p(y, x \mid \theta)^{\beta} \pi(\theta)^{\beta} d \theta$. Note that the ratio actually does not depend on $\theta^{(i)}$. If the candidate path $y$ is not accepted, then a new parameter $\theta^{(i)}$ from $p_{\beta}\left(\theta \mid y^{(i)}, x\right)$ and a new path $y$ from the distribution $p_{\beta}\left(y \mid \theta^{(i)}, x\right)$ will be generated. At the end of the sampling, the path with highest probability is found:

$$
\hat{v}_{\mathrm{SA}}:=\arg \max _{k=1, \ldots, r ; i=1, \ldots, n_{\beta_{k}}} p\left(y_{\beta_{k}}^{(i)} \mid x\right) .
$$

Iterative conditional mode algorithm. As already mentioned, sampling from $p(y \mid x)$ is in general not possible even if the model is simple. Since for any path $y$ the probability $p(y \mid x)$ can be found, then also for any site $t$ the probability $p_{t}\left(y_{t} \mid y_{-t}, x\right)$ can be calculated, where $p_{t}\left(y_{t} \mid y_{-t}, x\right)$ stands for the probability of observing $y_{t}$ at site $t$ given the rest of the sequence and $x$. Note that because $p(y \mid x)$ is not a Markov measure, $p_{t}\left(y_{t} \mid y_{-t}, x\right)$ is not necessarily the same as $p_{t}\left(y_{t} \mid y_{t-1}, y_{t+1}, x\right)$. The iterative conditional mode $(I C M)$ updates paths iteratively as follows. It starts from a sequence $y^{(0)}$. To obtain $y^{(i+1)}$, the sequence $y^{(i)}$ is updated site-by-site by the following rule:

$$
y_{t}^{(i+1)}=\arg \max _{k \in S} p_{t}\left(k \mid y_{1}^{(i+1)}, \ldots, y_{t-1}^{(i+1)}, y_{t+1}^{(i)}, \ldots, y_{n}^{(i)}, x\right) .
$$

Thus, the ICM algorithm acts similarly to single site sampling ([6], [13]), but instead of generating a random state, at every step it picks a state with maximum probability. In [9], the ICM algorithm is used under the name 'greedy algorithm'. It is indeed greedy in the sense that the update of every site increases the probability $p(y \mid x)$. The ICM algorithm converges when no further changes occur in the estimated sequence; the output will be denoted by $\hat{v}_{\mathrm{ICM}}$.

It is well known from the theory of simulated annealing that such a greedy update can cause the output to be trapped in a local maximum (see, e.g. [40]), and our numerical examples confirm that. However, since [9] is one of the few papers that considers segmentation in the Bayesian framework by non-stochastic methods, we include this method in our study.

\section{Numerical examples}

To illustrate the behaviour of the segmentation methods described in Section 2, we will present the results of two examples. In the first example we study the case with known emission distributions and transition 
probabilities following Dirichlet priors. Thus, $\theta=\theta_{t r}, \pi=\pi_{t r}$ and under $\pi$, the rows of the transition matrix are independent with the $l$-th row having a Dirichlet distribution $\operatorname{Dir}\left(\alpha_{l 1}, \cdots, \alpha_{l 4}\right)$. In the second example emission parameters also are assumed to be unknown and normal emissions with conjugate priors are studied. Since the estimation criterion is $\arg \max _{y} p(y \mid x)$, the main measure of goodness is $p(y \mid x)$ or equivalently, $\ln p(y, x)$. We will also study how the methods perform in regard to initial state sequences and how the estimated state paths depend on different sets of prior parameters.

\subsection{General framework}

The data is generated from an HMM with four underlying states, thus $S=\{1,2,3,4\}$. The emission distributions are normal with common variance $\sigma^{2}=0.25$, the emission distribution corresponding to state $k$ is $\mathcal{N}\left(\mu_{k}, \sigma^{2}\right)$ with $\mu_{1}=-0.7, \mu_{2}=0, \mu_{3}=0.7$ and $\mu_{4}=1.4$, respectively. The transition matrix is given by $\mathbb{P}=\left(p_{l j}\right)$ with $p_{l l}=0.6, l=1, \ldots, 4$, and $p_{l j}=0.4 / 3$, otherwise. The initial distribution $\left(p_{0 k}\right)$ is given by $p_{0 k}=0.25, k=1,2,3,4$. The length of the generated data sequence $x$ is $n=600$.

Hyperparameters. Recall that we use the parametrization $\alpha_{l j}=M q_{l j}, l, j=1, \ldots, 4$, where $Q=\left(q_{l j}\right)$ is a transition matrix and $M>0$ the precision parameter. We will consider three $Q$-matrices:

$$
Q_{1}=\left(q_{l j}\right) \text { with } q_{l j}=0.25 \forall l, j ; \quad Q_{2}=\mathbb{P} ; \quad Q_{3}=\left(q_{l j}\right) \text { with } q_{l l}=0.4, q_{l j}=0.2 \text { for } l \neq j .
$$

Thus, the combination $Q_{1}$ and $M=4$ corresponds to uniform priors on transition parameters, and $Q_{1}$ together with very large $M$ puts a uniform prior $p(y)$ on sequences (see also Section 4.1). The matrices $Q_{2}$ and $Q_{3}$ favour sequences with long blocks; the smaller $M$ is, the more such behavior is pronounced. To explain our choices of $M$ in simulations, let us give some intuition about the role of $M$ in some procedures. First, the Bayesian EM updates (7.4) for this parametrization are given by

$$
p_{l j}^{(i+1)}=\frac{\xi^{(i)}(l, j)+\left(M q_{l j}-1\right)}{\sum_{j} \xi^{(i)}(l, j)+(M-K)},
$$

where $\xi^{(i)}(l, j)$ is the expected number of transitions from state $l$ to $j$ at iteration $i$, which varies between 0 and $n-1$. If all transitions are equally likely, with our $n=600$ it is approximatively of order 37 . If $M$ is much larger than $n$, then the influence of data in (3.1) is negligible and the output of the procedure is very close to $Q$. On the other hand, a necessary condition in (3.1) is that $M q_{l j}>1$, which gives a lower bound to $M$. A similar argument holds for segmentation EM. Since for any integer $n$ large enough (see e.g. [17]), $\psi(n) \approx \ln (n-0.5)$, where $\gamma \approx 0.577$, we can for large $n \ll m$ use the approximation $\psi(m)-\psi(n) \approx \ln (m-0.5)-\ln (n-0.5)$. Disregarding the fact that $M q_{l j}$ might not be an integer, (2.3) gives that for a given state sequence $y$,

$$
u_{l j}(y) \approx \frac{M q_{l j}+n_{l j}(y)-0.5}{M+n_{l}(y)-0.5}
$$

If $M$ is very small in comparison to $n_{l j}$, then $u_{l j} \approx \frac{n_{l j}(y)}{n_{l}(y)}$ and the segmentation EM algorithm is practically the same as the segmentation MM algorithm. If on the other hand $M$ is too big, then the data are negligible and the output is close to the Viterbi path with $Q$. Based on these arguments, we consider the following constants $M: 600,150,50,10,5$. Observe that segmentation MM and Bayesian EM are applicable when $M q_{l j}>1$, which is restrictive when hyperparameters $\alpha_{l j} \leq 1$ are of interest. 
Initial sequences. Since the non-stochastic methods studied here depend on initial path values, the choice of initial paths has an important role in our numerical examples. All our procedures are designed to start with initial sequence, but a closer inspection of formulae (2.3), (7.1) and (7.5) reveals that when emission parameters are known and transition probabilities have Dirichlet priors, then the segmentation EM, segmentation MM, Bayesian EM and variational Bayes algorithms actually depend on $y^{(0)}$ only through the frequency matrix or empirical transition matrix $\left(n_{l j}\left(y^{(0)}\right)\right)$. The only deterministic algorithm that uses the full initial sequence as information and not only its summary measure through the number of empirical transitions is ICM. Therefore, it is expected that ICM is more sensitive with respect to initial sequences, because there are many more actual sequences than frequency matrices. For MCMC methods such as simulated annealing the initial value does not matter, because the number of sweeps is typically large.

Since our goal is to find the global maximum of $p(y \mid x)$ and the output of a method depends typically on the frequency matrix of the initial sequence, we try to choose initial sequences so that the corresponding frequency matrices will be different. Theoretically we would somehow like to cover the whole space of transition matrices. In the simplest case - that is, for a two-state model - we could for example choose transition matrices as follows:

$$
\left(\begin{array}{cc}
p & 1-p \\
1-q & q
\end{array}\right), \quad \text { where } \quad p, q \in\{0.25,0.5,0.75\}
$$

This would provide us with nine different transition matrices which could then be used to generate random sequences as realizations of a Markov chain with initial distribution being the stationary one. In the case of four states applying the described approach becomes more complicated. Therefore, in our examples we have considered 15 transition matrices $B_{1}, \ldots, B_{15}$ for generating initial sequences, which are obtained as follows. The first three matrices are just our $Q_{1}, Q_{2}$ and $Q_{3}$. The rest, $B_{4}, \ldots, B_{15}$, have been randomly generated: each row of $B_{l}$ has been independently generated from $\operatorname{Dir}(\alpha, \alpha, \alpha, \alpha)$, where the following 12 constants $\alpha=(0.3,0.5,0.7,0.8,0.9,1,1.1,1.2,1.3,1.5,1.7,1.9)$ have been used. From each matrix we have generated three random sequences as realizations of a Markov chain with initial distribution being the stationary one. We study also the initial sequence $y^{(0)}$ that corresponds to maximizing emissions pointwise, that is

$$
y_{t}^{(0)}=\arg \max _{k=1,2,3,4} f_{k}\left(x_{t}\right), \quad t=1, \ldots, n,
$$

which was suggested in [9]. For given $Q$, a good candidate for initial path is always the Viterbi path obtained using $Q$, therefore the last initial sequence considered is the Viterbi path obtained with the transition matrix $Q$. Thus all together we have studied 47 initial sequences. Given a set of hyperparameters and a non-stochastic iterative method, every initial sequence produces an output sequence. The maximum number of different output sequences is 47 . The smaller that number, the more robust or less sensitive with respect to initial sequences the method is. The end result of the method is given by the best output sequence, i.e. the one that has the largest $\log$-likelihood $\ln p(y, x)$. Since the emission densities $f_{k}$, $k=1, \ldots, K$, are fixed, the optimality criterion $\ln p(y, x)$ can for a given state path $y$ be calculated as

$$
\ln p(x, y)=\ln p(y)+\ln p(x \mid y)=\ln p(y)+\sum_{t=1}^{n} \ln f_{y_{t}}\left(x_{t}\right),
$$

where $\ln p(y)$ is as in (1.6). 


\subsection{Example 1: fixed emission distributions}

The main purpose of the first example is to compare the general performance of the algorithms. All non-stochastic methods (segmentation EM, segmentation MM, VB, ICM, Bayesian EM, standard EM) were run with all 47 initial sequences, whereas for simulated annealing one initial sequence was used. In the case of segmentation EM, segmentation MM and ICM the algorithm stopped when there were no further changes in the estimated state sequence. In simulated annealing a cooling schedule with inverse temperatures equally spaced in the range $[1,10.2]$ was used, where for every inverse temperature 15 paths were generated. Observe that for $Q_{2}$ and $Q_{3}$ segmentation MM and Bayesian EM were not applicable for $M=5\left(M q_{l j}>1\right.$ is not fulfilled for all $\left.q_{l j}\right)$, therefore the respective cell values of the tables summarizing the results for different methods are 'na'.

In Table 1 , for every method the best $\log$-likelihood value $\ln p(\hat{v}, x)$ over the outcomes corresponding to 47 initial sequences is presented, where $\hat{v}$ denotes the best output sequence for the corresponding method. The number in the brackets gives the number of different outputs out of 47 possible. The best log-likelihood value over all the methods for each set of hyperparameters is given in bold. As the table shows, the best results are generally obtained by segmentation EM and segmentation MM. The results for those methods differ for five sets of hyperparameters, and then sometimes the segmentation EM performs slightly better and sometimes the other way around. The similarity of the segmentation EM and MM methods is explained in Section 5. In Table 1, we can also see that VB and Bayesian EM behave quite similarly; this will also be clarified in Section 5. Observe that Bayesian EM is independent of initial sequence, while VB can result in different path outcomes. We can see that for EM-type methods the number of different outputs (sensitivity) increases when $M$ decreases and this makes sense, because a smaller $M$ means that data has more influence. Notice that ICM is the most sensitive among the studied methods, resulting in a different outcome for basically every initial sequence. It can also be remarked that the number of different outputs in the table for segmentation EM and segmentation MM shows that the initial state sequences generated from the same transition matrix result often in different output sequences.

The log-likelihood values in Table 1 give a general summary measure for comparing the best paths over the methods. To understand better how different these best paths really are, we have counted the pointwise differences in comparison to the best path and summarized these in Table 2. If the best state path over all the methods is $\hat{v}$ and the best path for a method we want to compare it with is $y$, then the sum of pointwise differences is given by $\sum_{t=1}^{n} I\left\{\hat{v}_{t} \neq y_{t}\right\}$. We can see that in the worst case, the path estimates can differ from the best path in up to $1 / 3$ of the path points, see VB and Bayesian EM for $Q_{1}$ and $M=50$.

Tables 1 and 2 summarize the results of different methods for a fixed observation sequence $x$. To observe the general behavior of the algorithms, we have rerun these simulations for 20 different observation sequences. Different observation sequences show a similar pattern to that in Table 1. We now study how segmentation EM and segmentation MM perform in comparison to simulated annealing. Table 3 summarizes the results for our 15 sets of hyperparameters and 20 observation sequences. The counts in the first half of the table (columns $\mathrm{SA}_{\max }, \mathrm{sEM}_{\max }, \mathrm{sMM}_{\max }$ ) present for each set of hyperparameters the number of best scores over 20 observation sequences. Each time a method is counted as best when it reaches the maximum log-likelihood for a given set of hyperparameters. Thus, for example, if all the three methods resulted in the same state path estimate, every method is counted as the best or 'winner'. In our example we can conclude that EM-type methods perform better than simulated annealing, since simulated annealing does not give the maximum log-likelihood value as often as EM-type methods. The second part of the table (columns $\mathrm{SA}_{\text {min }}, \mathrm{sEM}_{\text {min }}, \mathrm{sMM}_{\text {min }}$ ) presents for each method and for a given 


\begin{tabular}{|c|c|c|c|c|c|c|c|c|}
\hline$Q$ & $M$ & sEM & sMM & $\mathrm{ICM}$ & $\mathrm{VB}$ & $\mathrm{B}(\mathrm{EM})$ & EM & $\mathrm{SA}$ \\
\hline \multirow[t]{5}{*}{$\overline{\overline{Q_{1}}}$} & 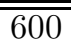 & "-1071.76(6) &  & -1071.76 (11) & $\overline{c-1072.93(2)}$ & -1072.98 & 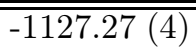 & -1072.02 \\
\hline & 150 & $-1017.57(30)$ & $-1017.59(27)$ & $-1030.48(46)$ & $-1051.82(4)$ & -1051.92 & $-1038.00(4)$ & -1031.63 \\
\hline & 50 & $-940.46(31)$ & $\mathbf{- 9 4 0 . 4 2}(31)$ & $-971.08(46)$ & $-1019.84(8)$ & -1019.07 & $-955.78(4)$ & -945.68 \\
\hline & 10 & $-861.81(38)$ & $-861.84(37)$ & $-909.67(46)$ & $-932.17(4)$ & -924.27 & $-878.13(4)$ & -865.10 \\
\hline & 5 & $-842.27(33)$ & $-842.30(35)$ & $-901.81(46)$ & $-909.12(1)$ & -899.53 & $-863.33(4)$ & -860.88 \\
\hline \multirow[t]{5}{*}{$Q_{2}$} & 600 & $-898.31(1)$ & $-898.31(1)$ & $-898.78(47)$ & $-899.28(1)$ & -899.28 & $-927.24(4)$ & -898.31 \\
\hline & 150 & $-882.51(8)$ & -882.51(7) & $-887.10(47)$ & $-888.32(2)$ & -887.35 & $-901.07(4)$ & -882.68 \\
\hline & 50 & $-862.31(18)$ & -862.31 (19) & $-877.19(47)$ & $-878.32(4)$ & -877.91 & $-876.46(4)$ & -865.63 \\
\hline & 10 & $-831.71(32)$ & $-831.71(36)$ & $-873.11(47)$ & $-871.52(3)$ & -869.18 & $-853.36(4)$ & -851.10 \\
\hline & 5 & $\mathbf{- 8 2 5 . 0 7}(36)$ & na & $-875.43(47)$ & $-870.10(2)$ & na & $-849.63(4)$ & -834.38 \\
\hline \multirow[t]{5}{*}{$\overline{Q_{3}}$} & 600 & $\mathbf{- 9 8 5 . 9 1 ( 6 )}$ & $\mathbf{- 9 8 5 . 9 1 ( 6 )}$ & $-988.46(47)$ & $-989.51(1)$ & -989.22 & $-1010.59(4)$ & -985.91 \\
\hline & 150 & $\mathbf{- 9 4 5 . 9 3}(13)$ & $-945.93(14)$ & $-961.60(47)$ & $-966.80(4)$ & -966.80 & $-964.31(4)$ & -946.58 \\
\hline & 50 & $-901.42(24)$ & $\mathbf{- 9 0 1 . 3 8}(27)$ & $-936.78(47)$ & $-940.26(2)$ & -938.31 & $-913.28(4)$ & -905.46 \\
\hline & 10 & $-846.64(34)$ & $\mathbf{- 8 4 6 . 6 4}(32)$ & $-901.30(47)$ & $-895.44(1)$ & -892.72 & $-865.09(4)$ & -865.05 \\
\hline & 5 & -833.49 (34) & na & $-895.74(47)$ & $-888.02(4)$ & na & $-855.95(4)$ & -843.05 \\
\hline
\end{tabular}

Table 1: The best $\log$-likelihood value $\ln p(x, \hat{v})$ (calculated as in (3.2)) obtained for every method in Example 1. The best result(s) for every set of hyperparameters is presented in bold. In the brackets, the number of different output sequences out of 47 possible is given.

\begin{tabular}{|c|r||r|r|r|r|r|r|r|}
\hline$Q$ & $M$ & sEM & sMM & ICM & VB & B(EM) & EM & SA \\
\hline \hline$Q_{1}$ & 600 & 0 & 0 & 0 & 19 & 20 & 167 & 10 \\
& 150 & 0 & 5 & 75 & 178 & 177 & 133 & 51 \\
& 50 & 6 & 0 & 119 & 198 & 198 & 108 & 24 \\
& 10 & 0 & 1 & 135 & 158 & 150 & 79 & 114 \\
& 5 & 0 & 2 & 151 & 141 & 135 & 81 & 87 \\
\hline$Q_{2}$ & 600 & 0 & 0 & 12 & 19 & 19 & 117 & 0 \\
& 150 & 0 & 0 & 60 & 53 & 47 & 117 & 6 \\
& 50 & 0 & 0 & 92 & 66 & 67 & 101 & 42 \\
& 10 & 0 & 0 & 170 & 113 & 110 & 81 & 86 \\
& 5 & 0 & na & 170 & 118 & na & 81 & 28 \\
\hline$Q_{3}$ & 600 & 0 & 0 & 38 & 41 & 37 & 90 & 9 \\
& 150 & 0 & 0 & 85 & 92 & 92 & 82 & 15 \\
& 50 & 7 & 0 & 130 & 125 & 122 & 83 & 163 \\
& 10 & 0 & 0 & 160 & 138 & 132 & 80 & 44 \\
& 5 & 0 & na & 151 & 129 & na & 81 & 54 \\
\hline
\end{tabular}

Table 2: Comparison of the estimated state sequences with the best Viterbi path estimate for each set of hyperparameters in Example 1. The number of pointwise differences compared to the best path estimate is presented.

set of hyperparameters the count over 20 observation sequences of when this method was strictly worse than the other two. Now we want to identify a 'loser', therefore we have counted how many times the respective method performs worst of the three methods according to the log-likelihood value. Here we can see that simulated annealing gives the lowest log-likelihood value most often. Thus, our example 
demonstrates that for a given cooling schedule and given set of initial state paths, EM-type algorithms perform actually better than simulated annealing.

\begin{tabular}{|c|c||c|c|c||c|c|c|}
\hline$Q$ & $M$ & $\mathrm{SA}_{\max }$ & $\mathrm{sEM}_{\max }$ & $\mathrm{sMM}_{\max }$ & $\mathrm{SA}_{\min }$ & $\mathrm{sEM}_{\min }$ & $\mathrm{sMM}_{\min }$ \\
\hline \hline$Q_{1}$ & 600 & 0 & 19 & 20 & 20 & 0 & 0 \\
& 150 & 0 & 18 & 15 & 20 & 0 & 0 \\
& 50 & 0 & 16 & 16 & 20 & 0 & 0 \\
& 10 & 1 & 14 & 14 & 18 & 1 & 0 \\
& 5 & 5 & 11 & 12 & 14 & 2 & 2 \\
\hline$Q_{2}$ & 600 & 16 & 20 & 20 & 4 & 0 & 0 \\
& 150 & 1 & 18 & 20 & 19 & 0 & 0 \\
& 50 & 2 & 16 & 15 & 18 & 1 & 1 \\
& 10 & 4 & 11 & 17 & 15 & 2 & 0 \\
& 5 & 4 & 16 & na & 16 & 4 & na \\
\hline$Q_{3}$ & 600 & 6 & 17 & 18 & 13 & 1 & 0 \\
& 150 & 0 & 19 & 20 & 20 & 0 & 0 \\
& 50 & 1 & 17 & 16 & 19 & 0 & 0 \\
& 10 & 5 & 13 & 11 & 14 & 1 & 4 \\
& 5 & 3 & 17 & na & 17 & 3 & na \\
\hline
\end{tabular}

Table 3: The counts over the Viterbi path estimates corresponding to 20 different observation sequences showing when the simulated annealing, segmentation EM and segmentation MM methods reached the maximum and minimum values of log-likelihood in Example 1. The minimum count shows how many times the respective method performs worst of the three methods according to the log-likelihood value.

\subsection{Example 2: priors on transition probabilities and emission parameters}

In the second example we assume that the parameters of emission densities are also unknown. The transition probabilities are modeled with Dirichlet priors as before. For emissions we consider normal distributions with conjugate prior distributions. The emission distribution corresponding to state $k$ is $\mathcal{N}\left(\mu_{k}, \sigma_{k}^{2}\right)$, where prior distributions for $\mu_{k}$ and $\sigma_{k}^{2}$ are given by a normal and inverse chi-square distribution respectively (also known as NIX priors):

$$
\pi_{e m}\left(\theta_{\mathrm{em}}\right)=\prod_{k=1}^{K} \pi_{e m}\left(\theta_{\mathrm{em}}^{k}\right), \quad \pi_{e m}\left(\theta_{\mathrm{em}}^{k}\right)=\pi\left(\mu_{k}, \sigma_{k}\right)=\pi\left(\sigma_{k}^{2}\right) \pi\left(\mu_{k} \mid \sigma_{k}^{2}\right),
$$

where

$$
\mu_{k} \mid \sigma_{k}^{2} \sim \mathcal{N}\left(\xi_{k}, \frac{\sigma_{k}^{2}}{\kappa_{0}}\right), \quad \sigma_{k}^{2} \sim \operatorname{Inv}-\chi^{2}\left(\nu_{0}, \tau_{0}^{2}\right)
$$

Here $\kappa_{0}, \nu_{0}$ and $\tau_{0}^{2}$ are hyperparameters that might depend on $k$, but in our example we assume they are equal. The calculations have been performed using the same 20 observation sequences $x$ and the same 47 initial sequences $y^{(0)}$ as in Example 1. We will also refer to this example as the Dirichlet-NIX case. The necessary formulae and computational details about the algorithms needed for the Dirichlet-NIX example can be found in the Appendix. As previously, for any path $y$ the universal optimality criterion 
is $\ln p(x, y)=\ln p(y)+\ln p(x \mid y)$, where $p(y)$ is calculated by (1.6) and under a NIX-prior $\ln p(x \mid y)$ is calculated as in (7.12).

In the Dirichlet-NIX example, the choice of emission hyperparameters affects segmentation results strongly, see also Subsection 4.2. The hyperparameters we consider are as follows: $\xi=(-0.7,0,0.7,1.4)$, $\tau_{0}^{2}=0.25, \kappa_{0}=10, \nu_{0}=50$. In simulated annealing a cooling schedule with inverse temperatures equally spaced in the range $[1,21]$ was used, for every inverse temperature 15 paths were generated. Again, for $Q_{2}$ and $Q_{3}$ segmentation MM and Bayesian EM were not applicable for $M=5\left(M q_{l j}>1\right.$ is not fulfilled for all $q_{l j}$ ), therefore the respective cell values of the tables summarizing the results for different methods are 'na'.

Since we are very much interested in how much faster non-stochastic methods perform computationally in comparison to MCMC methods, we start with presenting in Table 4 a summary of the behaviour of log-likelihood values over the 20 sequences and our 15 sets of transition hyperparameters just as in Table 3. The counts in Table 4 show that simulated annealing often gives the maximum log-likelihood value for $M=600$, otherwise EM-type algorithms perform generally better.

In Table 5 , the log-likelihood values $\ln p(\hat{v}, x)$ of the best path estimates for each method are presented for the same observation sequence as in Table 1. Again, the number of different outcome sequences out of 47 possible can be seen in the brackets. In general Table 5 shows the same pattern as Table 1: the best methods are segmentation MM and segmentation EM and they both outperform VB and Bayesian EM. For this observation sequence, the log-likelihood values for segmentation MM are slightly better than those for segmentation EM. But this is not a rule: the results for the other 19 observation sequences show that sometimes segmentation EM is better, and sometimes the other way around.

The log-likelihood values in Table 5 give again a general summary measure for comparing the best paths over the studied methods. Relatively small differences in log-likelihood values can incorporate large pointwise differences in the respective sequences. For example, the log-likelihood values of the best state sequences for segmentation EM and segmentation MM when $Q=Q_{2}$ and $M=50$ are -855.26 and -854.91, respectively. The pointwise difference between the state paths is 163 , the transition frequency matrices are given by

$$
\left(\begin{array}{cccc}
44 & 1 & 2 & 12 \\
0 & 333 & 0 & 16 \\
2 & 0 & 5 & 0 \\
14 & 15 & 0 & 155
\end{array}\right), \quad\left(\begin{array}{cccc}
37 & 3 & 0 & 4 \\
2 & 485 & 0 & 6 \\
0 & 0 & 0 & 0 \\
5 & 6 & 0 & 51
\end{array}\right) \text {. }
$$

\section{The role of hyperparameters in Bayesian segmentation}

In this section we will point out some important issues regarding the choice of hyperparameters which might be helpful also for interpretation of segmentation results.

\subsection{Dirichlet priors}

Uniform Dirichlet priors. Let us briefly discuss the case when $\alpha_{l j}=1$ for every $l$ and $j$. Then the rows of the transition matrix are uniformly distributed and therefore, the priors with $\alpha_{l j}=1$ are considered to be non-informative, which corresponds to not assuming anything of the transition matrix. In other words, all transition matrices are equiprobable and the expected values of all entries in the transition matrix are $\frac{1}{K}$. This might suggest that the same holds in the sequence space and no particular path structure (like sequences with long blocks or rapid changes) is preferred. But this is not the case 


\begin{tabular}{|c|c||c|c|c||c|c|c|}
\hline$Q$ & $M$ & $\mathrm{SA}_{\max }$ & $\mathrm{sEM}_{\max }$ & $\mathrm{sMM}_{\max }$ & $\mathrm{SA}_{\min }$ & $\mathrm{sEM}_{\min }$ & $\mathrm{sMM}_{\min }$ \\
\hline \hline$Q_{1}$ & 600 & 18 & 12 & 11 & 1 & 3 & 6 \\
& 150 & 12 & 9 & 8 & 7 & 6 & 5 \\
& 50 & 2 & 15 & 12 & 18 & 0 & 0 \\
& 10 & 5 & 12 & 11 & 15 & 2 & 0 \\
& 5 & 5 & 11 & 12 & 15 & 1 & 0 \\
\hline$Q_{2}$ & 600 & 17 & 4 & 6 & 3 & 7 & 2 \\
& 150 & 4 & 10 & 12 & 15 & 3 & 1 \\
& 50 & 1 & 10 & 14 & 19 & 0 & 1 \\
& 10 & 0 & 9 & 14 & 17 & 1 & 2 \\
& 5 & 4 & 16 & na & 16 & 4 & na \\
\hline$Q_{3}$ & 600 & 20 & 11 & 13 & 0 & 4 & 1 \\
& 150 & 7 & 13 & 10 & 13 & 1 & 2 \\
& 50 & 1 & 14 & 13 & 18 & 1 & 1 \\
& 10 & 3 & 10 & 14 & 16 & 2 & 1 \\
& 5 & 5 & 15 & na & 15 & 5 & na \\
\hline
\end{tabular}

Table 4: The counts over the Viterbi path estimates corresponding to 20 different observation sequences showing when the simulated annealing, segmentation EM and segmentation MM methods reached the maximum and minimum values of log-likelihood in Example 2. The minimum count shows how many times the respective method performed worst of the three methods according to the log-likelihood value.

\begin{tabular}{|c|c||c|c|c|c|c|c|c|}
\hline$Q$ & $M$ & sEM & sMM & ICM & VB & B(EM) & EM & SA \\
\hline \hline$Q_{1}$ & 600 & $-984.19(34)$ & $\mathbf{- 9 8 4 . 1 9 ( 3 3 )}$ & $-984.19(33)$ & $-988.38(8)$ & -988.33 & $-1129.93(35)$ & -984.19 \\
& 150 & $-964.23(25)$ & $-964.18(25)$ & $-964.17(45)$ & $-975.41(4)$ & -974.82 & $-1031.09(35)$ & $\mathbf{- 9 6 3 . 8 0}$ \\
& 50 & $-933.55(22)$ & $\mathbf{- 9 3 3 . 4 7 ( 2 3 )}$ & $-938.50(45)$ & $-964.38(2)$ & -964.28 & $-950.33(35)$ & -936.15 \\
& 10 & $\mathbf{- 8 5 4 . 6 9 ( 2 0 )}$ & $\mathbf{- 8 5 4 . 6 9}(24)$ & $\mathbf{- 8 5 4 . 6 9}(46)$ & $-915.08(1)$ & -910.94 & $-881.74(35)$ & -857.87 \\
& 5 & $\mathbf{- 8 3 9 . 8 9 ( 2 0 )}$ & $\mathbf{- 8 3 9 . 8 9}(25)$ & $\mathbf{- 8 3 9 . 8 9}(46)$ & $-890.10(9)$ & -887.64 & $-869.43(35)$ & -860.79 \\
\hline$Q_{2}$ & 600 & $-891.57(12)$ & $\mathbf{- 8 9 1 . 5 7 ( 1 0 )}$ & $-895.73(47)$ & $-900.09(1)$ & -898.46 & $-927.46(35)$ & $\mathbf{- 8 9 1 . 5 3}$ \\
& 150 & $\mathbf{- 8 8 1 . 3 6 ( 1 5 )}$ & $\mathbf{- 8 8 1 . 3 6 ( 1 7 )}$ & $-884.86(47)$ & $-887.66(1)$ & -887.26 & $-900.19(35)$ & -881.37 \\
& 50 & $-855.26(14)$ & $\mathbf{- 8 5 4 . 9 1}(16)$ & $-875.71(47)$ & $-873.85(1)$ & -874.64 & $-877.69(35)$ & -866.92 \\
& 10 & $-826.88(20)$ & $\mathbf{- 8 2 2 . 1 8 ( 2 9 )}$ & $-857.62(47)$ & $-864.01(1)$ & -858.24 & $-859.59(35)$ & -841.45 \\
& 5 & $\mathbf{- 8 1 8 . 9 5 ( 2 4 )}$ & na & $-841.14(47)$ & $-857.46(1)$ & na & $-857.55(35)$ & -841.52 \\
\hline$Q_{3}$ & 600 & $-938.34(22)$ & $\mathbf{- 9 3 8 . 1 2 ( 2 3 )}$ & $-938.34(47)$ & $-954.98(2)$ & -950.91 & $-1014.17(35)$ & $\mathbf{- 9 3 8 . 0 8}$ \\
& 150 & $\mathbf{- 9 2 7 . 6 2}(17)$ & $\mathbf{- 9 2 7 . 6 2}(18)$ & $-935.46(47)$ & $-936.39(4)$ & -936.62 & $-958.13(35)$ & -929.32 \\
& 50 & $-897.59(20)$ & $\mathbf{- 8 9 7 . 1 4}(18)$ & $-908.92(47)$ & $-918.83(2)$ & -919.05 & $-910.96(35)$ & -905.39 \\
& 10 & $-840.84(21)$ & $\mathbf{- 8 3 4 . 1 9}(24)$ & $-857.11(47)$ & $-888.23(4)$ & -883.58 & $-869.77(35)$ & -857.09 \\
& 5 & $\mathbf{- 8 2 6 . 5 7 ( 2 1 )}$ & na & $-841.30(47)$ & $-873.73(1)$ & na & $-862.78(35)$ & -849.91 \\
\hline
\end{tabular}

Table 5: The best $\log$-likelihood value $\ln p(x, \hat{v})$ (calculated with formulas (1.6) and (7.12)) for segmentation EM, segmentation MM, ICM, variational Bayes, Bayesian EM, standard EM and simulated annealing methods in Example 2. The best result(s) for every set of hyperparameters is presented in bold. The number of different output sequences out of 47 possible is given in the brackets.

- with uniform Dirichlet priors the state sequences are far from being equiprobable and the ones having long blocks are preferred. The following proposition proves that sequences with maximum prior weight 
are the constant ones.

Proposition 4.1. Let $\alpha_{l j}=1$ for every $l, j$. Then

$$
\arg \max _{y} p(y)=\left\{(i, \ldots, i), \quad i=\arg \max _{i} p_{0 i}\right\}
$$

Proof. When $\alpha_{l j}=1$, then $p(y)$ is according to (1.6) for any sequence $y$ given by

$$
p(y)=p_{0 y_{1}}[\Gamma(K)]^{K} \prod_{l} \frac{\prod_{j} \Gamma\left(1+n_{l j}(y)\right)}{\Gamma\left(K+n_{l}(y)\right)}=p_{0 y_{1}}[\Gamma(K)]^{K} \prod_{l} \frac{\prod_{j} n_{l j}(y) !}{\left(n_{l}(y)+K-1\right) !} .
$$

For the proof it suffices to show that any constant sequence maximizes the product term in the expression above. Fix $y$ and denote $n_{l j}:=n_{l j}(y)$. Since for every $l=1, \ldots, K, \sum_{j} n_{l j}=n_{l}$ and $\sum_{l} n_{l}=n-1$, the following inequality holds for any integer $a>0$ : $\prod_{l=1}^{K}\left(n_{l}+a\right) \geq a^{K-1}(n-1+a)$, where the equality holds only if $n_{l}=n-1$ for some $l=1, \ldots, K$. Therefore,

$$
\prod_{l=1}^{K} \frac{\prod_{j=1}^{K} n_{l j} !}{\left(n_{l}+K-1\right) !} \leq \prod_{l=1}^{K} \frac{n_{l} !}{\left(n_{l}+K-1\right) !} \leq\left(\frac{1}{2 \cdot 3 \cdots(K-1))}\right)^{(K-1)}(n(n+1) \cdots(n+K-2))^{-1} .
$$

The inequality is strict if $\exists j, k$ such that $n_{j}>0$ and $n_{k}>0$. Thus, the upper bound is reached only if $n_{l}=n-1$ for some $l=1, \ldots, K$, that is we have a constant state sequence.

To summarize: assuming nothing about the transition matrix is not equivalent to not assuming anything about the state sequences. On the contrary, equiprobable paths correspond to the fixed transition matrix with all entries equal to $\frac{1}{K}$, which is a very specific and strong assumption about the transition matrix.

The role of precision parameter. Recall the parametrization $\alpha_{l j}=M q_{l j}$. When $q_{l j}=1 / K$ for every $l$ and $j$, then the precision parameter $M$ can be considered as a regularization parameter in the optimization problem

$$
\max _{y}\left(\ln p(x \mid y)+\ln p_{M}(y)\right),
$$

where the subscript $M$ denotes the dependence on $M$. Increasing $M$ corresponds to reducing the role of $\ln p(y)$, thus the limit case $M \rightarrow \infty$ corresponds to $\ln p(y)=$ const (all paths are equiprobable). Therefore, when $M \rightarrow \infty$, (4.1) reduces to $\max _{y} p(x \mid y)$. The case with $M=K$ corresponds to the case of uniform Dirichlet priors with $\alpha_{l j}=1$, and in this case the role of $\ln p(y)$ in (4.1) is to make the output sequences more constant. Thus, when $q_{l j}=1 / K$, then decreasing $M$ means changing the sequence prior $p_{M}(y)$ so that the sequences with large blocks will have more weight.

In this article, we also consider $Q$-matrices, where the entries on the main diagonal have larger values than the off-diagonal elements. With such $Q$, for every $M$ the sequence prior $p_{M}(y)$ puts more weight on the sequences with big blocks and the most probable sequences are constant ones. However, this behavior is even more pronounced for small $M$. Indeed, if $M \rightarrow \infty$, then for every $y, p_{M}(y) \rightarrow p_{0 y_{1}} \prod_{l, j} q_{l j}^{n_{l j}(y)}:=$ $p_{\infty}(y)$. It is easy to see that for constant sequences the convergence is monotone. For example, if $y=1, \ldots, 1$, then as $M \rightarrow \infty$, it holds that $p_{M}(y) \searrow p_{01} q_{11}^{n-1}=p_{\infty}(y)$. Thus, since the entries on the main diagonal have larger values than the off-diagonal elements, the limit measure $p_{\infty}$ puts more weight on sequences with large blocks. But due to the monotone convergence, we see that for smaller $M$ the measure $p_{M}(y)$ concentrates on such sequences even more. 


\subsection{Clustering under normal emissions with NIX priors}

To understand the role of emission hyperparameters, it is instructive to consider the optimization problem $\max _{y} p(x \mid y)$. In the Bayesian HMM setup this corresponds to the limit case $M \rightarrow \infty$ when $q_{l j}=1 / K$ $\forall l, j$, thus $p_{M}(y)=$ const. Since $p_{M}(y)$ is not involved in segmentation anymore, the whole temporal structure of the model is dropped and it is more correct to refer to the problem as clustering. We will show that the nature of the clustering problem and its solutions depend heavily on the hyperparameters. It turns out that under NIX priors, the family of possible clustering problems is large, including many familiar $k$-means related problems. We will briefly discuss some of them. Typically, 'standard' problems are obtained when the hyperparameters $\nu_{0}, \kappa_{0}$ and $\tau_{0}^{2}$ approach their extreme values, that is 0 or $\infty$. The details about the formulae are given in the Appendix.

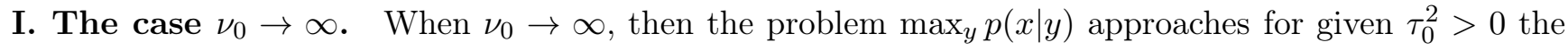
following clustering problem: find clusters $S_{1}, \ldots, S_{K}$ that minimize

$$
\sum_{k=1}^{K}\left[\sum_{t \in S_{k}}\left(x_{t}-\bar{x}_{k}\right)^{2}+\frac{\kappa_{0} m_{k}}{\kappa_{0}+m_{k}}\left(\bar{x}_{k}-\xi_{k}\right)^{2}+\tau_{0}^{2} \ln \left(\kappa_{0}+m_{k}\right)\right]
$$

which is equivalent to minimizing

$$
\sum_{k=1}^{K} \min _{\mu_{k} \in \mathcal{X}}\left[\sum_{t \in S_{k}}\left(x_{t}-\mu_{k}\right)^{2}+\kappa_{0}\left(\mu_{k}-\xi_{k}\right)^{2}+\tau_{0}^{2} \ln \left(\kappa_{0}+m_{k}\right)\right],
$$

where $m_{k}=\left|S_{k}\right|$ (see the Appendix). The first term in (4.3) corresponds to the sum of least squares, the second term tries to form clusters around $\xi_{k}$ and the third term tries to build clusters of unequal size. Thus, if $\tau_{0}^{2}$ is very big, then one cluster gets very big and the others are empty or very small. For small $\tau_{0}^{2}$, the influence of the third term is small. When $\kappa_{0} \rightarrow 0$, the second term disappears. This corresponds to the case where the variance of $\mu_{k}$ is infinite (uninformative prior for $\mu_{k}$ ). The case with $\kappa_{0} \rightarrow 0$ and $\tau_{0}^{2} \rightarrow 0$ corresponds to the classical $k$-means optimization problem.

The segmentation MM algorithm acts in the case $\nu_{0} \rightarrow \infty$ for any $\tau_{0}^{2}$ as follows: given clusters $S_{1}^{(i)}, \ldots, S_{K}^{(i)}$, find the corresponding cluster centres

$$
\mu_{k}^{(i)}=\left(m_{k}^{(i)} \bar{x}_{k}^{(i)}+\kappa_{0} \xi_{k}\right) /\left(\kappa_{0}+m_{k}^{(i)}\right) .
$$

Given these centres, find new clusters corresponding to the Voronoi partition:

$$
S_{k}^{(i+1)}=\left\{x_{t}:\left|x_{t}-\mu_{k}^{(i)}\right|=\min _{l}\left|x_{t}-\mu_{l}^{(i)}\right|\right\} .
$$

In the case of segmentation EM algorithm the cluster centres $\mu_{k}^{(i)}$ are calculated in the same way as for segmentation MM, but the clustering rule is different:

$$
S_{k}^{(i+1)}=\left\{x_{t}:\left(x_{t}-\mu_{k}^{(i)}\right)^{2}+\frac{\tau_{0}^{2}}{m_{k}^{(i)}+\kappa_{0}}=\min _{l}\left[\left(x_{t}-\mu_{l}^{(i)}\right)^{2}+\frac{\tau_{0}^{2}}{m_{l}^{(i)}+\kappa_{0}}\right]\right\} .
$$

The term $\tau_{0}^{2} /\left(m_{l}^{(i)}+\kappa_{0}\right)$ in (4.4) affects cluster size. When $\tau_{0}^{2}$ is small, then segmentation EM and MM give the same result, but when $\tau_{0}^{2}$ increases, then segmentation EM tends to produce clusters of unequal size, 
whereas segmentation MM remains unaffected by $\tau_{0}^{2}$. When $\kappa_{0} \rightarrow 0$ and $\tau_{0}^{2} \rightarrow 0$, then both algorithms converge to the standard Lloyd algorithm.

When $\kappa_{0} \rightarrow \infty$, the clustering problem in (4.2) reduces to minimizing $\sum_{k=1}^{K} \sum_{t \in S_{k}}\left(x_{t}-\xi_{k}\right)^{2}$ with the solution given by $S_{k}=\left\{x_{t}:\left|x_{t}-\xi_{k}\right|=\min _{l}\left|x_{t}-\xi_{l}\right|\right\}$. The solution matches fully with intuition, because $\nu_{0} \rightarrow \infty$ and $\kappa_{0} \rightarrow \infty$ corresponds to the case with fixed normal emissions with means $\xi_{k}$ and variances $\tau_{0}^{2}$, thus clustering is trivial.

II. The case with finite $\nu_{0}$. For a given $\nu_{0}$, the general optimization problem we have is the following: find clusters $S_{1}, \ldots, S_{k}$ minimizing the sum

$$
-\sum_{k} \ln \Gamma\left(\frac{\nu_{0}+m_{k}}{2}\right)+\frac{1}{2} \sum_{k} \ln \left(\kappa_{0}+m_{k}\right)+\sum_{k} \frac{\nu_{0}+m_{k}}{2} \min _{\mu_{k} \in \mathcal{X}} \ln \left(\nu_{0} \tau_{0}^{2}+\sum_{t \in S_{k}}\left(x_{t}-\mu_{k}\right)^{2}+\kappa_{0}\left(\mu_{k}-\xi_{k}\right)^{2}\right) .
$$

The first two terms in (4.5) tend to make size of the clusters unequal. This follows from the observation that under the constraint $\sum_{k} m_{k}=n$, the products

$$
\prod_{k=1}^{K} \Gamma\left(\frac{\nu_{0}+m_{k}}{2}\right), \quad \prod_{k=1}^{K}\left(\kappa_{0}+m_{k}\right)^{-\frac{1}{2}}
$$

are maximized when $m_{k}=n$ holds for some $k$. The smaller $\nu_{0}$ and $\kappa_{0}$ are, the bigger is the influence of the first two terms. When $\kappa_{0} \rightarrow \infty$, the problem of finding optimal clusters $S_{1}, \ldots, S_{K}$ reduces to minimizing

$$
-\sum_{k} \ln \Gamma\left(\frac{\nu_{0}+m_{k}}{2}\right)+\sum_{k} \frac{\nu_{0}+m_{k}}{2} \ln \left(\nu_{0} \tau_{0}^{2}+\sum_{t \in S_{k}}\left(x_{t}-\xi_{k}\right)^{2}\right)
$$

The solution to this problem gives bigger clusters than obtained by minimizing $\sum_{k=1}^{K} \sum_{t \in S_{k}}\left(x_{t}-\xi_{k}\right)^{2}$. When $\tau_{0}^{2} \rightarrow \infty$, then the last term in (4.5) disappears and the problem reduces to finding clusters that minimize the sum of the first two terms in (4.5). The solution here is one big cluster.

As our examples have shown, clustering under NIX setting is highly dependent on hyperparameters, and the choice of hyperparameters can strongly affect the resulting segmentation.

\section{Similarity of the algorithms studied}

We have seen that out of the five non-stochastic optimization methods (segmentation EM, segmentation MM, ICM, Bayesian EM and VB), ICM is clearly most inadequate, because it depends heavily on initial sequences and gets stuck in local optima. The other four methods can be divided into two groups, which can be characterized as segmentation-based methods (segmentation EM and segmentation MM) and parameter-based methods (Bayesian EM and VB). We call VB a parameter-based method, because it updates the parameters iteratively and then, with final $h_{k}$ and $u_{l j}$, the Viterbi algorithm is applied (see Subsection 7.1). The segmentation EM and MM methods apply the Viterbi algorithm at each iteration step. Our numerical examples demonstrate a clear advantage of the segmentation-based methods, which is also expected, because segmentation EM optimizes the objective function of interest and segmentation MM behaves very similarly.

We already observed the pairwise similarity of the segmentation-based methods and the parameterbased methods in Examples 1 and 2. In the case that emission distributions are known, the four algorithms 
can be further summarized as follows. Comparing (3.1) and (7.8) shows that both the Bayesian EM and VB updates can be written as

$$
\ln p_{l j}^{*(i+1)}=f_{1}\left(\xi^{(i)}(l, j)+M q_{l j}\right)-f_{2}\left(\sum_{j} \xi^{(i)}(l, j)+M\right),
$$

where $p_{l j}^{*}$ is either $p_{l j}$ (Bayesian EM) or $u_{l j}(\mathrm{VB})$, and where $f_{1}(x)=\ln (x-1), f_{2}(x)=\ln (x-K)$ for Bayesian EM and $f_{1}=f_{2}=\psi$ for VB. Similarly, the transition updates for segmentation MM (7.1) and segmentation EM (2.3) can be written as

$$
\ln p_{l j}^{*(i+1)}=f_{1}\left(n_{l j}\left(y^{(i)}\right)+M q_{l j}\right)-f_{2}\left(n_{l}\left(y^{(i)}\right)+M\right),
$$

where $f_{1}(x)=\ln (x-1), f_{2}(x)=\ln (x-K)$ for segmentation MM and $f_{1}=f_{2}=\psi$ for segmentation EM. Thus, the four methods can be characterized by two parameters: the function parameter (ln vs $\psi$ ) and the counts parameter (direct counts $n_{l j}(s)$ versus averaged counts $\xi(l, j)$ ):

\begin{tabular}{|l|c|c|}
\hline Counts/Function & $\ln$ & $\psi$ \\
\hline Direct $\left(n_{l j}\right)$ & sMM & sEM \\
\hline Averaged $(\xi(l, j))$ & $\mathrm{B}(\mathrm{EM})$ & $\mathrm{VB}$ \\
\hline
\end{tabular}

The results of Examples 1 and 2 show that the difference in functions does not influence the algorithm as much as the difference in counts, because the methods behave similarly row-wise. The examples also show that in terms of maximizing the main study criterion, that is the posterior likelihood, the methods using direct counts outperform the methods that use averaged counts. We have noticed that the methods using ln-function give sometimes slightly larger posterior probability than the ones using $\psi$, and this is a matter for future research.

\section{Conclusions and further research}

The paper is mainly devoted to studying non-stochastic algorithms for finding the Viterbi path in Bayesian hidden Markov models. The performance of the segmentation EM method introduced in the article has been compared with other well-known non-stochastic methods (segmentation MM, iterative conditional mode, variational Bayes, Bayesian EM) as well as with the simulated annealing approach.

The segmentation EM method that optimizes the correct objective function mostly outperforms the other studied methods, often also the simulated annealing method. It should be noted that the possibility to apply the segmentation EM algorithm should not be taken for granted for any model. For many models the EM algorithm can be written down easily theoretically, but the maximization and/or expectation step can be impractically complicated to perform. One example of such a model is the hidden Markov model with infinite state space (hierarchical Dirichlet processes), where the E-step involves intractable integrals. In our setup with Dirichlet prior distributions and emissions from the exponential family the E-step involves well-known digamma functions and the M-step reduces to the Viterbi algorithm, therefore the segmentation EM is easily applicable.

Our study demonstrates that when the main goal of inference is segmentation, then the Bayesian approach should be used. The Bayesian setup enables to concentrate directly on segmentation and skip the parameter estimation step.

It is a little surprising that the segmentation MM method behaves in our examples as well as the segmentation EM algorithm, since the performance of the same method in the context of parameter 
estimation (known then as Viterbi training) is often notoriously bad. The similarity of the segmentation EM and MM methods is shortly discussed in Section 5, but a good performance of segmentation MM needs further investigation. For practitioners we advise to be careful with the segmentation MM method, because it does not optimize the right criterion function as segmentation EM does.

The segmentation EM and MM methods are sensitive with respect to initial sequences, therefore the choice of initial sequences is crucial. Since for both algorithms it is actually the empirical transition matrix of the initial sequence that is the input to the algorithm, initial sequences should be chosen so that the corresponding empirical transition matrices are different and somehow cover the search space.

The article brings out the important role of hyperparameters in the Bayesian context, different issues regarding this topic are thoroughly discussed in Section 4. Our results demonstrate that hyperparameters determine largely the nature of the segmentation problem and the properties of the solution, they also control the influence of data. The simulation examples show that even a small change in some of the hyperparameters can change the problem drastically. This is obviously a disappointment for practitioners because the idea of Bayesian approach is to get rid off the choice of parameters, and now it turns out that the hyperparameters should be chosen equally carefully. It seems to us that the role of hyperparameters is overlooked in the literature, at least in the segmentation context.

The concluded research opens several interesting directions for future studies. As pointed out in Introduction, a common alternative to the Viterbi path in practice is the PMAP path, which is the state path estimate that minimizes the expected number of classification errors. For given parameters (known or estimated), the PMAP path can be found with the well-known forward-backward algorithm. How to find the PMAP path in the Bayesian setup is an open and challenging question, since there is no obvious analogue to the segmentation EM or MM algorithm in this case.

Another appealing research question is about incorporating inhomogeneity to the model. In the Bayesian setup inhomogeneity means the change of priors from time to time. In the case of known change points and independent priors the situation reduces to cutting the whole model into independent submodels. However, in general and thus even in the Bayesian setup it might instead be preferable to consider the model where the change points are not exactly known. Suppose there are a few possible transition matrices $\left\{\mathbb{P}_{i}\right\}$ and the underlying chain $Y$ is inhomogeneous driven by one of these matrices at a time. However, we do not know a priori which matrix drives the transition at a given time $t$. An elegant way for incorporating such kind of variability and information into the model is the so-called triplet Markov models (TMMs) introduced by Piecynski [3]. In TMMs, instead of a Markov chain $Y$ a bivariate Markov chain $(Y, U)$ is considered, where the additional component $U$ allows a change of the transition matrix. Since $Y$ is not a Markov chain, the pair $(X, Y)$ is not an HMM anymore, and therefore it is not obvious how to find the Viterbi path in this model. A closer inspection indicates that segmentation EM might still be applicable, at least under some additional assumptions. A further step would be to consider a hierarchical model where the Dirichlet hyperparameters, say $\alpha$, are modeled in the way described, that is $(\alpha, U)$ is a bivariate Markov chain. This incorporates both the approach with Dirichlet transition priors and the approach with variable change points.

Since segmentation in the Bayesian setup heavily depends on hyperparameters, it would be tempting to put additional priors on hyperparameters. Such models are sometimes called hierarchical. Another example of a hierarchical model is hierachical Dirichlet processes (see [16], Ch. 5), where the number of hidden states is not fixed any more. Such models are complicated and how to design non-stochastic segmentation algorithms in this case is a very interesting research area. 


\section{Appendix}

\subsection{General formulae for the segmentation methods studied}

Due to our independence assumption, all emission and transition parameters can be estimated separately. In the formulae of this section we use the same notation for the random parameters $p_{l, j}, \mu_{k}$ and $\sigma_{k}^{2}$, $k, l, j \in\{1, \ldots, K\}$, and the corresponding estimates. The exact meaning can be understood from the context.

Segmentation MM. In the case of Dirichlet priors the matrix $\theta_{t r}^{(i+1)}$ can be found row-wise, the $l$-th row is the posterior mode:

$$
p_{l j}^{(i+1)}=\frac{\alpha_{l j}+n_{l j}\left(y^{(i)}\right)-1}{\alpha_{l}+n_{l}\left(y^{(i)}\right)-K} .
$$

Emission parameters can be updated independently:

$$
\theta_{e m}^{k,(i+1)}=\arg \max _{\theta_{e m}^{k}} p\left(\theta_{e m}^{k} \mid x_{S_{k}}\right)=\arg \max _{\theta_{e m}^{k}}\left[\sum_{t: y_{t}^{(i)}=k} \ln f_{k}\left(x_{t} \mid \theta_{e m}^{k}\right)+\ln \pi_{e m}^{k}\left(\theta_{e m}^{k}\right)\right], \quad k=1, \ldots, K,
$$

where $x_{S_{k}}$ is the subsample of $x$ corresponding to state $k$ in $y^{(i)}$. Formally, for every sequence $y \in S^{n}$ define $S_{k}(y)=\left\{t \in\{1, \ldots, n\}: y_{t}=k\right\}$, then $x_{S_{k}}=\left\{x_{t}: t \in S_{k}\right\}$.

Bayesian EM. The emission updates are given by

$$
\theta_{e m}^{k,(i+1)}=\arg \max _{\theta_{e m}^{k}}\left[\sum_{t} \ln f_{k}\left(x_{t} \mid \theta_{e m}^{k}\right) \gamma_{t}^{(i)}(k)+\ln \pi_{e m}^{k}\left(\theta_{e m}^{k}\right)\right], \quad k=1, \ldots, K,
$$

where

$$
\gamma_{t}^{(i)}(k):=P\left(Y_{t}=k \mid X=x, \theta^{(i)}\right)=\sum_{y: y_{t}=k} p\left(y \mid \theta^{(i)}, x\right) .
$$

In the case of Dirichlet priors the transition updates are given by

$$
p_{l j}^{(i+1)}=\frac{\xi^{(i)}(l, j)+\left(\alpha_{l j}-1\right)}{\sum_{j} \xi^{(i)}(l, j)+\left(\alpha_{l}-K\right)}, \quad \text { where } \quad \xi^{(i)}(l, j):=\sum_{t=1}^{n-1} P\left(Y_{t}=l, Y_{t+1}=j \mid x, \theta^{(i)}\right) .
$$

Since one of the studied methods (ICM) starts with an initial sequence, in order the comparison to be fair, we let all the other methods to start with a sequence as well. Therefore, for a given initial sequence $y^{(0)}$, define

$$
\gamma_{t}^{(0)}(k):=I_{k}\left(y_{t}^{(0)}\right), \quad \xi^{(0)}(l, j):=n_{l j}\left(y^{(0)}\right)
$$

Variational Bayes approach. Let us have a closer look at the measure $q_{Y}^{(i+1)}(y)$. We are going to show that there exists an $\operatorname{HMM}(Z, X)$ such that for every sequence $y, q_{Y}^{(i+1)}(y)=P(Z=y \mid X=x)$. By definition,

$$
q_{Y}^{(i+1)}(y) \propto \exp \left[\int \ln p(\theta, y \mid x) q_{\theta}^{(i+1)}(d \theta)\right] .
$$

Apply the notation from (2.2) in the current case:

$$
u_{l j}^{(i+1)}=\exp \left[\int \ln p_{l j}\left(\theta_{t r}\right) q_{\theta}^{(i+1)}(d \theta)\right], \quad h_{k}^{(i+1)}\left(x_{t}\right)=\exp \left[\int \ln f_{k}\left(x_{t} \mid \theta_{e m}^{k}\right) q_{\theta}^{(i+1)}(d \theta)\right] .
$$


Since $\ln p(\theta, y \mid x)=\ln \pi(\theta)+\ln p(y, x \mid \theta)-\ln p(x)$, we obtain

$$
\begin{aligned}
& \int \ln p(\theta, y \mid x) q_{\theta}^{(i+1)}(d \theta)=\int \ln \pi(\theta) q_{\theta}^{(i+1)}(d \theta)-\ln p(x)+\int \ln p(y, x \mid \theta) q_{\theta}^{(i+1)}(d \theta) \\
& =c\left(q_{\theta}^{(i+1)}, x\right)+\ln p_{0 y_{1}}+\sum_{l j} n_{l j}(y) \ln u_{l j}^{(i+1)}+\sum_{k=1}^{K} \sum_{t: y_{t}=k} \ln h_{k}^{(i+1)}\left(x_{t}\right) \\
& =c\left(q_{\theta}^{(i+1)}, x\right)+\ln p_{0 y_{1}}+\sum_{l j} n_{l j}(y) \ln \tilde{u}_{l j}^{(i+1)}+\sum_{k=1}^{K} \sum_{t: y_{t}=k} \ln \tilde{h}_{k}^{(i+1)}\left(x_{t}\right),
\end{aligned}
$$

where $\tilde{u}_{l j}$ is the normalized quantity, $\tilde{u}_{l j}:=\frac{u_{l j}}{\sum_{j} u_{l j}}$, and $\tilde{h}_{k}\left(x_{t}\right):=\left(\sum_{j} u_{k j}\right) h_{k}\left(x_{t}\right)$, if $t \leq n-1, \tilde{h}_{k}\left(x_{n}\right)=$ $h_{k}\left(x_{n}\right)$. Let now $(Z, X)$ be an HMM, where $Z$ is the underlying Markov chain with transition matrix $\left(\tilde{u}_{l j}\right)$ and emission densities are given by $\tilde{h}_{k}$. From (7.6) it follows that $q_{Y}^{(i+1)}(y) \propto P(Z=y \mid X=x)$. Since $q_{Y}^{(i+1)}$ and $P(Z \in \cdot \mid X=x)$ are both probability measures, it follows that they are equal. To stress the dependence on iterations, we will denote $q_{Y}^{(i+1)}(y)=P^{(i+1)}(Z=y \mid X=x)$.

Let us now calculate $q_{\theta}$. Let $\gamma_{t}^{(i)}(k)$ denote the marginal of $q_{Y}^{(i)}(y)$,

$$
\gamma_{t}^{(i)}(k):=P^{(i)}\left(Z_{t}=k \mid X=x\right)=\sum_{y: y_{t}=k} q_{Y}^{(i)}(y)
$$

Observe that

$$
\sum_{y} \ln p(y, x \mid \theta) q_{Y}^{(i)}(y)=C_{1}+\sum_{l, j} \ln p_{l j}\left(\theta_{t r}\right)\left(\sum_{y} n_{l j}(y) q_{Y}^{(i)}(y)\right)+\sum_{t=1}^{n} \sum_{k=1}^{K} \ln f_{k}\left(x_{t} \mid \theta_{e m}^{k}\right) \gamma_{t}^{(i)}(k),
$$

where $C_{1}:=\sum_{k}\left(\ln p_{0 k}\right) \gamma_{1}^{(i)}(k)$. The sum $\sum_{y} n_{l j}(y) q_{Y}^{(i)}(y)$ is the expected number of transitions from $l$ to $j$, so that using the equality $q_{Y}^{(i)}(y)=P^{(i)}(Z=y \mid X=x)$, we have

$$
\sum_{y} n_{l j}(y) q_{Y}^{(i)}(y)=\sum_{t=1}^{n-1} P^{(i)}\left(Z_{t}=l, Z_{t+1}=j \mid X=x\right)=: \xi^{(i)}(l, j) .
$$

Therefore,

$$
\ln q_{\theta}^{(i+1)}(\theta)=C+\ln \pi_{t r}\left(\theta_{t r}\right)+\ln \pi_{e m}\left(\theta_{e m}\right)+\sum_{l, j} \xi^{(i)}(l, j) \ln p_{l j}\left(\theta_{t r}\right)+\sum_{t=1}^{n} \sum_{k=1}^{K} \ln f_{k}\left(x_{t} \mid \theta_{e m}^{k}\right) \gamma_{t}^{(i)}(k) .
$$

From (7.7) we can see that under $q_{\theta}^{(i+1)}$ the parameters $\theta_{t r}, \theta_{e m}^{1}, \ldots, \theta_{e m}^{K}$ are still independent and can therefore be updated separately. In the case of Dirichlet transition priors the rows are independent as well. The transition update for the $l$-th row and the emission update for the $k$-th component are given by

$$
q_{\theta}^{(i+1)}\left(p_{l 1}, \ldots, p_{l K}\right) \propto \prod_{j=1}^{K} p_{l j}^{\alpha_{l j}-1+\xi^{(i)}(l, j)}, \quad q_{\theta}^{(i+1)}\left(\theta_{e m}^{k}\right) \propto \pi\left(\theta_{e m}^{k}\right) \prod_{t=1}^{n}\left(f_{k}\left(x_{t} \mid \theta_{e m}^{k}\right)\right)^{\gamma_{t}^{(i)}(k)} .
$$

The whole VB approach is applicable since $\xi^{(i)}(l, j)$ and $\gamma_{t}^{(i)}(k)$ can be found by the standard forwardbackward formulae using $\tilde{u}_{l j}^{(i)}$ and $\tilde{h}_{k}^{(i)}$. Actually, it is not difficult to see that in these formulae the original 
$u_{l j}^{(i)}$ and $h_{k}^{(i)}$ can be used instead of the standardized ones. To summarize, in our setup the VB approach yields the following algorithm for calculating $\hat{v}_{\mathrm{VB}}$. For a given initial sequence $y^{(0)}$, find vector $\gamma_{t}^{(0)}$ and matrix $\xi^{(0)}$ as in (7.5). Given $\gamma_{t}^{(i)}$ and $\xi^{(i)}$, update $u_{l j}^{(i+1)}$ and $h_{k}^{(i+1)}$ as follows:

$$
\begin{gathered}
u_{l j}^{(i+1)}=\exp \left[\psi\left(\alpha_{l j}+\xi^{(i)}(l, j)\right)-\psi\left(\alpha_{l}+\xi^{(i)}(l)\right)\right], \quad \text { where } \quad \xi^{(i)}(l):=\sum_{j} \xi^{(i)}(l, j), \\
h_{k}^{(i+1)}\left(x_{t}\right)=\exp \left[\int \ln f_{k}\left(x_{t} \mid \theta_{e m}^{k}\right) q_{\theta}^{(i+1)}(d \theta)\right], \quad \text { where } \quad q_{\theta}^{(i+1)}\left(\theta_{e m}^{k}\right) \propto \pi\left(\theta_{e m}^{k}\right) \prod_{t=1}^{n}\left(f_{k}\left(x_{t} \mid \theta_{e m}^{k}\right)\right)^{\gamma_{t}^{(i)}(k)} .
\end{gathered}
$$

With these parameters, $\xi^{(i+1)}$ and $\gamma_{t}^{(i+1)}$ can be calculated with the usual forward-backward procedure for HMM. Then update $u_{l j}^{(i+2)}$ and $h_{k}^{(i+2)}$ and so on. After the convergence, say after $m$ steps, apply the Viterbi algorithm with transitions $\left(u_{i j}^{(m)}\right)$ and emission densities $h_{k}^{(m)}$. The obtained path maximizes $q_{Y}^{(m)}(y)$ over all the paths, so it is $\hat{v}_{\mathrm{VB}}$.

Simulated annealing. Because of independence of the emission and transition parameters, it holds even for $\beta>1$ that $p_{\beta}(\theta \mid y, x)=p_{\beta}\left(\theta_{t r} \mid y\right) p_{\beta}\left(\theta_{e m} \mid y, x\right)$, thus the transition and emission parameters can be sampled separately. When the rows of a transition matrix have independent Dirichlet priors, the $l$-th row can be generated from the Dirichlet distribution with parameters $\beta\left(n_{l k}(s)+\alpha_{l k}\right)+1-\beta, k=1, \ldots, K$. For given $\theta$, sampling from $p(y \mid \theta, x)$ can be performed in various ways: we use so-called Markovian Backward Sampling (Algorithm 6.1.1 in [6]). To sample from $p_{\beta}(y \mid \theta, x)$, note that

$$
p(x, y \mid \theta)^{\beta}=\frac{p_{0 y_{1}}^{\beta}}{\sum_{j} p_{0, j}^{\beta}} \prod_{t=2}^{n} \tilde{p}_{y_{t-1} y_{t}} \tilde{f}_{y_{t}}\left(x_{t}\right),
$$

where $\tilde{p}_{i j}:=p_{i j}^{\beta} / \sum_{j} p_{i j}^{\beta}$, and $\tilde{f}_{k}\left(x_{t}\right):=\left(\sum_{j} p_{i j}^{\beta}\right) f_{k}^{\beta}\left(x_{t}\right), t=1, \ldots, n-1, \tilde{f}_{k}\left(x_{n}\right):=\left(\sum_{j} p_{0 j}^{\beta}\right) f_{k}^{\beta}\left(x_{n}\right)$. Although the functions $\tilde{f}_{k}$ are not densities, one can still use Markovian Backward Sampling.

\subsection{Non-stochastic segmentation algorithms for the Dirichlet-NIX case}

Suppose the emission distribution corresponding to state $k$ is $\mathcal{N}\left(\mu_{k}, \sigma_{k}^{2}\right)$, where prior distributions for $\mu_{k}$ and $\sigma_{k}^{2}$ are given by a normal and scaled inverse-chi-square distribution, respectively:

$$
\mu_{k} \mid \sigma_{k}^{2} \sim \mathcal{N}\left(\xi_{k}, \frac{\sigma_{k}^{2}}{\kappa_{0}}\right), \quad \sigma_{k}^{2} \sim \operatorname{Inv}-\chi^{2}\left(\nu_{0}, \tau_{0}^{2}\right)
$$

Here $\kappa_{0}, \nu_{0}$ and $\tau_{0}^{2}$ are hyperparameters that might depend on $k$, but in our example we assume they are equal. Recall the density of $\operatorname{Inv}-\chi^{2}\left(\nu, \tau^{2}\right)$ :

$$
f\left(x ; \nu, \tau^{2}\right)=\frac{\left(\tau^{2} \nu / 2\right)^{\nu / 2}}{\Gamma(\nu / 2)} x^{-(1+\nu / 2)} \exp \left[-\frac{\nu \tau^{2}}{2 x}\right] .
$$

If $X \sim \operatorname{Inv}-\chi^{2}\left(\nu, \tau^{2}\right)$, then

$$
E X=\frac{\tau^{2} \nu}{\nu-2}, \quad \operatorname{Var}(X)=\frac{2 \tau^{4} \nu^{2}}{(\nu-2)^{2}(\nu-4)}, \quad E(\ln X)=\ln \left(\frac{\nu \tau^{2}}{2}\right)-\psi\left(\frac{\nu}{2}\right), \quad E X^{-1}=\tau^{-2},
$$


and the mode of the distribution is given by $\nu \tau^{2} /(\nu+2)$. Therefore, if $\nu_{0}$ and $\kappa_{0}$ are both very large, then $\sigma_{k}^{2} \approx \tau_{0}^{2}$ and $\mu_{k} \approx \xi_{k}$, and we get back to the first example. If $\nu_{0}$ is very large, then $\sigma_{k}^{2} \approx \tau_{0}^{2}$, so that emission variances are $\tau_{0}^{2}$, but the variance of the mean is approximately $\tau_{0}^{2} / \kappa_{0}$.

Since emission and transition parameters are independent, the transition parameters can be updated as previously, that is as described in Section 7.1. Because the emission components $\left(\theta_{e m}^{1}, \ldots, \theta_{e m}^{K}\right)$ are independent under prior and posterior, it holds that $p\left(\theta_{e m} \mid y, x\right)=\prod_{k} p\left(\theta_{e m}^{k} \mid x_{S_{k}}\right)$, where $x_{S_{k}}$ is the subsample of $x$ along $y$ corresponding to state $k$. Let $m_{k}(y)$ be the size of $x_{S_{k}}$. Let $\bar{x}_{k}$ and $s_{k}^{2}$ be the mean and variance of $x_{S_{k}}$. Since NIX-priors are conjugate, for any state $k$ the posterior parameters $\kappa_{k}$, $\nu_{k}, \mu_{k}$ and $\tau_{k}^{2}$ can be calculated as follows:

$$
\begin{aligned}
\kappa_{k} & =\kappa_{0}+m_{k}, \quad \nu_{k}=\nu_{0}+m_{k}, \\
\mu_{k} & =\frac{\kappa_{0}}{\kappa_{0}+m_{k}} \xi_{k}+\frac{m_{k}}{\kappa_{0}+m_{k}} \bar{x}_{k}, \\
\nu_{k} \tau_{k}^{2} & =\nu_{0} \tau_{0}^{2}+\left(m_{k}-1\right) s_{k}^{2}+\frac{\kappa_{0} m_{k}}{\kappa_{0}+m_{k}}\left(\bar{x}_{k}-\xi_{k}\right)^{2}, \quad s_{k}^{2}=\frac{1}{m_{k}-1} \sum_{t \in S_{k}}\left(x_{t}-\bar{x}_{k}\right)^{2},
\end{aligned}
$$

see [32]. We also need to calculate for every path $y$ the joint probability $p(x, y)=p(y) p(x \mid y)$. Due to the independence of transition and emission parameters, $p(y)$ is still as in (1.6) and $p(x \mid y)$ depends on emission parameters, only. According to the formula for the marginal likelihood (see, e.g. [32]) we obtain

$$
p(x \mid y)=\prod_{k=1}^{K} \int \prod_{t \in S_{k}} f_{k}\left(x_{t} \mid \theta_{e m}^{k}\right) \pi\left(\theta_{e m}^{k}\right) d \theta_{e m}^{k}=\prod_{k=1}^{K} \frac{\Gamma\left(\frac{\nu_{k}}{2}\right)}{\Gamma\left(\frac{\nu_{0}}{2}\right)} \sqrt{\frac{\kappa_{0}}{\kappa_{k}}} \frac{\left(\nu_{0} \tau_{0}^{2}\right)^{\frac{\nu_{0}}{2}}}{\left(\nu_{k} \tau_{k}^{2}\right)^{\frac{\nu_{k}}{2}}} \pi^{-\frac{m_{k}}{2}} .
$$

We will now give a more detailed description of the non-stochastic algorithms for Example 2.

Bayesian EM. Start with initial state sequence $y^{(0)}$. With this sequence, find for any state $k$ the parameters $\kappa_{k}, \mu_{k}, \nu_{k}, \tau_{k}^{2}$ as defined in (7.9), (7.10), (7.11) and calculate the posterior modes, that is update

$$
p_{l j}^{(1)}=\frac{n_{l j}\left(y^{(0)}\right)+\left(\alpha_{l j}-1\right)}{\sum_{j} n_{l j}\left(y^{(0)}\right)+\left(\alpha_{l}-K\right)}, \quad \mu_{k}^{(1)}=\mu_{k}, \quad\left(\sigma_{k}^{2}\right)^{(1)}=\frac{\nu_{k} \tau_{k}^{2}}{\nu_{k}+2}, \quad k=1 \ldots, K .
$$

With these parameters calculate the vectors $\gamma_{t}^{(1)}$ and matrix $\left(\xi^{(1)}(l, j)\right)$ as in (7.3) and (7.4) using the forward-backward formulae. Given $\gamma_{t}^{(i)}$ and $\xi^{(i)}(l, j)$, the transition parameters are updated according to (7.4). The emission updates are given by (7.2). Let us calculate $\theta_{e m}^{k,(i+1)}$ for the NIX-model. Suppress $k$ from the notation and observe that $\theta_{e m}^{(i+1)}=\left(\mu^{(i+1)},\left(\sigma^{2}\right)^{(i+1)}\right)$ maximizes the following function over $\mu$ and $\sigma^{2}$ :

$$
\begin{aligned}
& \sum_{t} \ln f\left(x_{t} \mid \mu, \sigma^{2}\right) \gamma_{t}^{(i)}+\ln \pi\left(\mu \mid \sigma^{2}\right)+\ln \pi\left(\sigma^{2}\right)= \\
& \text { const }-\frac{1}{2}\left[\left(\ln \sigma^{2}\right)\left(\sum_{t} \gamma_{t}^{(i)}+\left(\nu_{0}+3\right)\right)+\frac{1}{\sigma^{2}}\left(\sum_{t}\left(x_{t}-\mu\right)^{2} \gamma_{t}^{(i)}+(\mu-\xi)^{2} \kappa_{0}+\nu_{0} \tau_{0}^{2}\right)\right] .
\end{aligned}
$$

The solutions $\mu_{k}^{(i+1)}$ and $\left(\sigma_{k}^{2}\right)^{(i+1)}$ are given by:

$$
\mu_{k}^{(i+1)}=\frac{\sum_{t} x_{t} \gamma_{t}^{(i)}(k)+\xi_{k} \kappa_{0}}{\sum_{t} \gamma_{t}^{(i)}(k)+\kappa_{0}}, \quad\left(\sigma_{k}^{2}\right)^{(i+1)}=\frac{\nu_{0} \tau_{0}^{2}+\sum_{t}\left(x_{t}-\mu_{k}^{(i+1)}\right)^{2} \gamma_{t}^{(i)}(k)+\left(\mu_{k}^{(i+1)}-\xi_{k}\right)^{2} \kappa_{0}}{\sum_{t} \gamma_{t}^{(i)}(k)+\nu_{0}+3} .
$$


With $\kappa_{0} \rightarrow 0$ (non-informative prior), $\mu_{k}^{(i+1)}$ is the same as in the standard EM algorithm. Using the updated parameters, calculate $\gamma_{t}^{(i+1)}$ and $\xi^{(i+1)}(l, j)$. Keep updating until the change in the log-likelihood is below the stopping criterion.

Segmentation EM. Given sequence $y^{(i)}$, calculate for every state $k$ the parameters $\kappa_{k}^{(i)}, \mu_{k}^{(i)}, \nu_{k}^{(i)}$ and $\left(\tau_{k}^{2}\right)^{(i)}$ using formulae (7.9), (7.10) and (7.11). With these parameters, calculate $h_{k}^{(i+1)}\left(x_{t}\right)$ as follows:

$\ln h_{k}^{(i+1)}\left(x_{t}\right)=-\frac{1}{2} \ln \left(2 \pi\left(\tau_{k}^{2}\right)^{(i)}\right)-\frac{1}{2}\left[\ln \left(\frac{\nu_{k}^{(i)}}{2}\right)-\psi\left(\frac{\nu_{k}^{(i)}}{2}\right)\right]-\frac{x_{t}^{2}}{2\left(\tau_{k}^{2}\right)^{(i)}}+x_{t} \frac{\mu_{k}^{(i)}}{\left(\tau_{k}^{2}\right)^{(i)}}-\frac{1}{2}\left[\frac{1}{\kappa_{k}^{(i)}}+\left(\frac{\mu_{k}^{(i)}}{\tau_{k}^{(i)}}\right)^{2}\right]$.

Compute the matrix $\left(u_{l j}^{(i+1)}\right)$, where $\ln u_{l j}^{(i+1)}=\psi\left(\alpha_{l j}+n_{l j}\left(y^{(i)}\right)\right)-\psi\left(\alpha_{l}+n_{l}\left(y^{(i)}\right)\right)$. To find $y^{(i+1)}$, apply the Viterbi algorithm with $u_{l j}^{(i+1)}$ and $h_{k}^{(i+1)}\left(x_{t}\right)$. Keep doing so until no changes occur in the path estimate.

Segmentation MM. Given $y^{(i)}$, calculate $\mu_{k}^{(i)}, \nu_{k}^{(i)}$ and $\left(\tau_{k}^{2}\right)^{(i)}$ using formulae (7.9), (7.10) and (7.11) and update the posterior modes as follows:

$$
p_{l j}^{(i+1)}=\frac{n_{l j}\left(y^{(i)}\right)+\left(\alpha_{l j}-1\right)}{\sum_{j} n_{l j}\left(y^{(i)}\right)+\left(\alpha_{l}-K\right)}, \quad \mu_{k}^{(i+1)}=\mu_{k}^{(i)}, \quad\left(\sigma_{k}^{2}\right)^{(i+1)}=\frac{\nu_{k}^{(i)}}{\nu_{k}^{(i)}+2}\left(\tau_{k}^{2}\right)^{(i)} .
$$

With these parameters find $y^{(i+1)}$ by the Viterbi algorithm. Keep doing so until no changes occur in the estimated state path.

VB algorithm. Given an initial state sequence $y^{(0)}$, find $h_{k}^{(1)}\left(x_{t}\right)$ and $u_{l j}^{(1)}$ as in the segmentation EM algorithm. With these parameters, calculate $\gamma_{t}^{(1)}$ and $\xi^{(1)}(l, j)$ using the forward-backward formulae. Given the matrix $\left(\xi^{(i)}(l, j)\right)$, update the matrix $\left(u_{l j}^{(i+1)}\right)$ according to $(7.8)$. Given $\gamma_{t}^{(i)}(k)$, the parameters $\kappa_{k}^{(i)}, \mu_{k}^{(i)}, \nu_{k}^{(i)}$ and $\left(\tau_{k}^{2}\right)^{(i)}$ can be calculated by (see, e.g., [30])

$$
\begin{aligned}
\kappa_{k}^{(i)} & =\kappa_{0}+g_{k}^{(i)}, \quad \nu_{k}^{(i)}=\nu_{0}+g_{k}^{(i)}, \quad g_{k}^{(i)}=\sum_{t=1}^{n} \gamma_{t}^{(i)}(k), \\
\mu_{k}^{(i)} & =\frac{\kappa_{0}}{\kappa_{0}+g_{k}^{(i)}} \xi_{k}+\frac{g_{k}^{(i)}}{\kappa_{0}+g_{k}^{(i)}} \tilde{x}_{k}^{(i)}, \quad \tilde{x}_{k}^{(i)}=\frac{1}{g_{k}^{(i)}} \sum_{t=1}^{n} \gamma_{t}^{(i)}(k) x_{t}, \\
\nu_{k}^{(i)}\left(\tau_{k}^{2}\right)^{(i)} & =\nu_{0} \tau_{0}^{2}+\sum_{t=1}^{n}\left(x_{t}-\tilde{x}_{k}^{(i)}\right)^{2} \gamma_{t}^{(i)}(k)+\frac{\kappa_{0} g_{k}^{(i)}}{\kappa_{0}+g_{k}^{(i)}}\left(\tilde{x}_{k}^{(i)}-\xi_{k}\right)^{2} .
\end{aligned}
$$

Compute then $h_{k}^{(i+1)}\left(x_{t}\right)$ as in (7.13). With help of $h_{k}^{(i+1)}\left(x_{t}\right)$ and $u_{l j}^{(i+1)}$, find $\gamma_{t}^{(i+1)}$ and $\xi^{(i+1)}(l, j)$ using the forward-backward formulae. After that update $h_{k}^{(i+2)}\left(x_{t}\right)$ and $u_{l j}^{(i+2)}$ and so on. When the VB algorithm has converged, say after $m$ steps, apply the Viterbi algorithm with $u_{l j}^{(m)}$ as transitions and with $h_{k}^{(m)}\left(x_{t}\right)$ as emission values. 


\subsection{Clustering formulae under normal emissions with NIX priors}

From (7.12) it follows that for any sequence $y^{\prime}$, the likelihood ratio is given by

$$
\frac{p(x \mid y)}{p\left(x \mid y^{\prime}\right)}=\prod_{k=1}^{K} \frac{\Gamma\left(\frac{\nu_{0}+m_{k}}{2}\right)}{\Gamma\left(\frac{\nu_{0}+m_{k}^{\prime}}{2}\right)} \prod_{k=1}^{K} \sqrt{\frac{\kappa_{0}+m_{k}^{\prime}}{\kappa_{0}+m_{k}}} \prod_{k=1}^{K} \frac{\left(\nu_{k}^{\prime} \tau_{k}^{\prime}\right)^{\frac{\nu_{0}}{2}}}{\left(\nu_{k} \tau_{k}^{2}\right)^{\frac{\nu_{0}}{2}}} \prod_{k=1}^{K} \frac{\left(\nu_{k}^{\prime} \tau_{k}^{\prime}\right)^{\frac{m_{k}^{\prime}}{2}}}{\left(\nu_{k} \tau_{k}^{2}\right)^{\frac{m_{k}}{2}}} .
$$

When $\nu_{0} \rightarrow \infty$ and $\tau_{0}^{2}>0$, then due to $\sum_{k} m_{k}=\sum_{k} m_{k}^{\prime}=n$ we have

$$
\lim _{\nu_{0} \rightarrow \infty} \prod_{k=1}^{K} \frac{\Gamma\left(\frac{\nu_{0}+m_{k}}{2}\right)}{\Gamma\left(\frac{\nu_{0}+m_{k}^{\prime}}{2}\right)}=1, \quad \lim _{\nu_{0} \rightarrow \infty} \prod_{k=1}^{K} \frac{\left(\nu_{k}^{\prime} \tau_{k}^{\prime}\right)^{\frac{m_{k}^{\prime}}{2}}}{\left(\nu_{k} \tau_{k}^{2}\right)^{\frac{m_{k}}{2}}}=1 .
$$

Write $\nu_{k} \tau_{k}^{2}$ as

$$
\nu_{k} \tau_{k}^{2}=\nu_{0} \tau_{0}^{2}+\sum_{t \in S_{k}}\left(x_{t}-\bar{x}_{k}\right)^{2}+\frac{\kappa_{0} m_{k}}{\kappa_{0}+m_{k}}\left(\bar{x}_{k}-\xi_{k}\right)^{2}=\nu_{0} \tau_{0}^{2}+A_{k}=\nu_{0} \tau_{0}^{2}\left(1+\frac{2 A_{k}}{2 \nu_{0} \tau_{0}^{2}}\right) .
$$

Then

$$
\frac{\left(\nu_{k}^{\prime} \tau_{k}^{\prime}\right)^{\frac{\nu_{0}}{2}}}{\left(\nu_{k} \tau_{k}^{2}\right)^{\frac{\nu_{0}}{2}}}=\frac{\left(1+\frac{2 A_{k}^{\prime}}{2 \nu_{0} \tau_{0}^{2}}\right)^{\frac{\nu_{0}}{2}}}{\left(1+\frac{2 A_{k}}{2 \nu_{0} \tau_{0}^{2}}\right)^{\frac{\nu_{0}}{2}}} \rightarrow \exp \left[\frac{A_{k}^{\prime}-A_{k}}{2 \tau_{0}^{2}}\right] .
$$

Therefore, when $\nu_{0} \rightarrow \infty$, then the likelihood ratio in (7.14) converges to

$$
\prod_{k=1}^{K} \sqrt{\frac{\kappa_{0}+m_{k}^{\prime}}{\kappa_{0}+m_{k}}} \exp \left[\frac{\sum_{k} A_{k}^{\prime}-\sum_{k} A_{k}}{2 \tau_{0}^{2}}\right] .
$$

Thus, maximizing $p(x \mid y)$ corresponds to the following clustering problem: find clusters $S_{1}, \ldots, S_{k}$ that minimize

$$
\sum_{k=1}^{K} \sum_{t \in S_{k}}\left(x_{t}-\bar{x}_{k}\right)^{2}+\kappa_{0} \sum_{k=1}^{K} \frac{m_{k}}{\kappa_{0}+m_{k}}\left(\bar{x}_{k}-\xi_{k}\right)^{2}+\tau_{0}^{2} \sum_{k=1}^{K} \ln \left(\kappa_{0}+m_{k}\right),
$$

which is formula (4.2). Given cluster $S_{k}$, it is easy to see that

$$
\arg \min _{\mu \in \mathbb{R}}\left[\sum_{t \in S_{k}}\left(x_{t}-\mu\right)^{2}+\kappa_{0}\left(\mu-\xi_{k}\right)^{2}\right]=\frac{m_{k} \bar{x}_{k}+\kappa_{0} \xi_{k}}{\kappa_{0}+m_{k}}=: \mu_{k}
$$

Since

$$
\sum_{t \in S_{k}}\left(x_{t}-\mu_{k}\right)^{2}+\kappa_{0}\left(\mu_{k}-\xi_{k}\right)^{2}=\sum_{t \in S_{k}}\left(x_{t}-\bar{x}_{k}\right)^{2}+\kappa_{0} \frac{m_{k}}{\kappa_{0}+m_{k}}\left(\bar{x}_{k}-\xi_{k}\right)^{2},
$$

we obtain (4.3).

To understand the behavior of the segmentation EM and segmentation MM algorithms when $\nu_{0} \rightarrow \infty$, recall the segmentation EM iteration formula from (7.13). When $\nu_{0} \rightarrow \infty$, then $\ln \left(\nu_{k}^{(i)} / 2\right)-\psi\left(\nu_{k}^{(i)} / 2\right) \rightarrow 0$ and $\left(\tau_{k}^{2}\right)^{(i)} \rightarrow \tau_{0}^{2}$. Thus, leaving the superscript $(i)$ out of the notation, we get

$$
\ln h_{k}\left(x_{t}\right) \rightarrow-\frac{1}{2} \ln \left(2 \pi\left(\tau_{0}^{2}\right)\right)-\frac{1}{2 \tau_{0}^{2}}\left(x_{t}-\mu_{k}\right)^{2}-\frac{1}{2\left(\kappa_{0}+m_{k}\right)},
$$

where $\mu_{k}$ is as in (7.15). The Viterbi alignment is now obtained as

$$
y_{t}=\arg \min _{k=1, \ldots, K}\left[\left(x_{t}-\mu_{k}\right)^{2}+\frac{\tau_{0}^{2}}{m_{k}+\kappa_{0}}\right] \text {. }
$$




\section{Acknowledgments}

This work is supported by Estonian institutional research funding IUT34-5.

\section{References}

[1] M. Beal. Variational algorithms for approximate Bayesian inference. PhD thesis, Gatsby Computational Neurosience unit, University College London, 2003.

[2] M. Beal and Z. Ghahramani. The variational Bayesian EM algorithm for incomplete data: with application to scoring graphical model structures. In Bayesian statistics, 7 (Tenerife, 2002), pages 453-463. Oxford Univ. Press, New York, 2003.

[3] D. Benboudjema and W. Piecynski. Unsupervised statistical of nonstastionary images using triplet markov fields. IEEE Transactions on Pattern Analysis and Machine Intelligence, 29(8):1367-1378, 2007.

[4] J. Besag. An introduction to Markov chain Monte Carlo methods. In Mathematical foundations of speech and language processing, volume 138 of IMA Vol. Math. Appl., pages 247-270. Springer, New York, 2004.

[5] C. Bishop. Pattern recognition and machine learning. Information Science and Statistics. Springer, New York, 2006.

[6] O. Cappé, E. Moulines, and T. Rydén. Inference in hidden Markov models. Springer, New York, 2005.

[7] L. Carvalho and C. Lawrence. Centroid estimation in discrete high-dimensional spaces with applications in biology. Proceedings of the National Academy of Sciences, 105(9):3209-3214, 2008.

[8] C. Christodoulopoulos, S. Goldwater, and M. Steedman. Two decades of unsupervised POS induction: How far have we come? In Proceedings of the Conference on Empirical Methods in Natural Language Processing, 2010.

[9] J. Corander, J. Xiong, Y. Cui, and T Koski. Optimal Viterbi Bayesian predictive classification for data from finite alphabets. J. Statist. Plann. Inference, 143(2):261-275, 2013.

[10] J.-B. Courbot, E. Monfrini, V. Mazet, and C. Collet. Oriented triplet Markov fields. Pattern Recognition Letters, 103:16-22, 2018.

[11] C. Fox and S. Roberts. A tutorial on variational Bayesian inference. Artificial intelligence review, $38(2): 85-95,2012$.

[12] K. Ganchev, B. Taskar, P. Fernando, and G. Joao. Posterior vs parameter sparsity in latent variable models. In Advances in Neural Information Processing Systems 22. 2009.

[13] J. Gao and M. Johnson. A comparison of Bayesian estimators for unsupervised hidden Markov model pos taggers. In Proceedings of the Conference on Empirical Methods in Natural Language Processing, EMNLP '08, pages 344-352, Stroudsburg, PA, USA, 2008. Association for Computational Linguistics. 
[14] S. Goldwater and T. Griffiths. A fully Bayesian approach to unsupervised part-of-speech tagging. In Proceedings of the 45th Annual Meeting of the Association for Computational Linguistics, Prague, Czech Republic, 2007.

[15] I. Gorynin, H. Gangloff, E. Monfrini, and W. Pieczynski. Assessing the segmentation performance of pairwise and triplet Markov models. Signal Processing, 145:183-192, 2018.

[16] N.L. Hjort, C. Holmes, P. Müller, and S.G. Walker, editors. Bayesian Nonparametrics. Cambridge University Press, New York, 2010.

[17] M. Johnson. Why doesn't EM find good HMM POS-taggers? In Proceedings of the 2007 Joint Conference on Empirical Methods in Natural Language Processing and Computational Natural Language Learning (EMNLP-CoNLL), pages 296-305, Prague, Czech Republic, June 2007. Association for Computational Linguistics.

[18] M. Jordan, Z. Gharhami, T. Jaakkola, and L. Saul. An introduction to variational methods for graphical models. Machine learning, 37:183-233, 1999.

[19] A. Koloydenko, M. Käärik, and J. Lember. On adjusted Viterbi training. Acta Appl. Math., 96(13):309-326, 2007.

[20] A. Koloydenko and J. Lember. Bridging Viterbi and posterior decoding: A generalized risk approach to hidden path inference based on hidden Markov models. Journal of Machine Learning Research, $15: 1-58,2014$.

[21] A. Koloydenko and J. Lember. Bridging Viterbi and posterior decoding: A generalized risk approach to hidden path inference based on hidden Markov models. Journal of Machine Learning Research, $15: 1-58,2014$.

[22] T. Koski. Hidden Markov Models for Bioinformatics, volume 2 of Computational Biology Series. Kluwer Academic Publishers, Dordrecht, 2001.

[23] P. Lanchantin, J. Lapuyade-Lahorgue, and W. Pieczynski. Unsupervised segmentation of triplet Markov chains hidden with long-memory noise. Signal Processing, 88(5):1134-1151, 2008.

[24] J. Lember and A. Koloydenko. Adjusted Viterbi training: A proof of concept. Probab. Eng. Inf. Sci., 21(3):451 - 475, 2007.

[25] J. Lember and A. Koloydenko. The Adjusted Viterbi training for hidden Markov models. Bernoulli, 14(1):180-206, 2008.

[26] J. Lember, K. Kuljus, and A. Koloydenko. Theory of segmentation. In P. Dymarsky, editor, Hidden Markov Models, Theory and Applications, pages 51-84. InTech, 2011.

[27] J. Marin and C. Robert. Bayesian core: a practical approach to computational Bayesian statistics. Springer Texts in Statistics. Springer, New York, 2007.

[28] A. Maruotti and R. Rocci. A mixed non-homogeneous hidden markov model for categorical data, with application to alcohol consumption. Statistics in Medicine, 31(9):871-886.

[29] A. Maruotti and T. Rydén. A semiparametric approach to hidden markov models under longitudinal observations. Statistics and Computing, 19:381-393, 2009. 
[30] C. A. McGrory and D. M. Titterington. Variational Bayesian analysis for hidden Markov models. Aust. N. Z. J. Stat., 51(2):227-244, 2009.

[31] G. McLachlan and T. Krishnan. The EM algorithm and extensions. Wiley, Hoboken, NJ, 2008.

[32] K. Murphy. Conjugate Bayesian analysis of the Gaussian distribution. Technical report, 2007.

[33] L. R. Rabiner. A tutorial on hidden Markov models and selected applications in speech recognition. Proc. IEEE, 77(2):257-286, 1989.

[34] T. Rydén. EM versus Markov chain Monte Carlo for estimation of hidden Markov models: a computational perspective. Bayesian Anal., 3(4):659-688, 2008.

[35] A. Samé, C. Ambroise, and G. Govaert. A classification EM algorithm for binned data. Comput. Statist. Data Anal., 51(2):466-480, 2006.

[36] A. Samé, C. Ambroise, and G. Govaert. An online classification EM algorithm based on the mixture model. Stat. Comput., 17(3):209-218, 2007.

[37] S. Scott. Bayesian methods for hidden Markov models: recursive computing in the 21st century. $J$. Amer. Statist. Assoc., 97(457):337-351, 2002.

[38] V. Smidl and A. Quinn. The Variational Bayes Method in Signal Processing. Springer, 2006.

[39] S. Watanabe. Algebraic geometry and statistical learning theory, volume 25 of Cambridge Monographs on Applied and Computational Mathematics. Cambridge University Press, Cambridge, 2009.

[40] G. Winkler. Image analysis, random fields and Markov chain Monte Carlo methods, volume 27 of Applications of Mathematics. Springer-Verlag, Berlin, 2003.

[41] C. Yau and C. Holmes. A decision-theoretic approach for segmental classification. Ann. Appl. Stat., 7(3):1814-1835, 2013. 